\title{
GLOBAL PARAMETER AND HELIOSEISMIC TESTS OF SOLAR VARIABILITY MODELS
}

\author{
L. H. Li, S. Basu, S. Sofia, F. J. Robinson, and P. Demarque \\ Department of Astronomy, Yale University, P.O. Box 208101, New Haven, CT 06520-8101 \\ AND \\ D. B. Guenther \\ Department of Astronomy and Physics, Saint Mary's University, Halifax, Nova Scotia B3H 3C3, Canada; \\ li@astro.yale.edu \\ Received 2002 June 17; accepted 2003 March 21
}

\begin{abstract}
We construct models of the structure and evolution of the Sun which include variable magnetic fields and turbulence. The magnetic effects are (1) magnetic pressure, (2) magnetic energy, and (3) magnetic modulation to turbulence. The effects of turbulence are (1) turbulent pressure, (2) turbulent kinetic energy, and (3) turbulent inhibition of the radiative energy loss of a convective eddy, and (4) turbulent generation of magnetic fields. Using these ingredients we construct five types of solar variability models (including the standard solar model) with magnetic effects. These models are in part based on three-dimensional numerical simulations of the superadiabatic layers near the surface of the Sun. The models are tested with several sets of observational data, namely, the changes of (1) the total solar irradiance, (2) the photospheric temperature, (3) radius, (4) the position of the convection zone base, and (5) low- and medium-degree solar oscillation frequencies. We find that turbulence plays a major role in solar variability, and only a model that includes a magnetically modulated turbulent mechanism can agree with all the current available observational data. We find that because of the somewhat poor quality of all observations (other than the helioseismological ones), we need all data sets in order to restrict the range of models.
\end{abstract}

Subject headings: Sun: activity — Sun: helioseismology — Sun: interior — Sun: oscillations

\section{INTRODUCTION}

Modern standard solar models are known to yield the solar structure to an amazing degree of precision (see, e.g., Basu, Pinsonneault, \& Bahcall 2000). These models, however, cannot explain the solar cycle and other solar cyclerelated variability, the reason being that these models do not include magnetic fields and related time variability. The aim of this paper is to construct models that can be used to model the solar activity cycle.

The cyclical variation in the number of sunspots is only one of the manifestations of the solar cycle. Satellite observations have revealed an $11 \mathrm{yr}$ cycle in the total solar irradiance (TSI) with an amplitude of about $0.1 \%$ that is in phase with the solar magnetic activity cycle (e.g., Wilson \& Hudson 1991; Fröhlich 2000). The effective temperature of the Sun also changes, and there is evidence that the solar radius changes too. Solar oscillation frequencies are also known to change with time in step with solar activity levels. Thus, any model constructed to try and model the solar activity cycle must explain all these changes - the changes in the global parameters, i.e., the irradiance, temperature, and radius, as well as changes in the oscillation frequencies.

The fact that the so-called solar constant is not actually constant raises the possibility of a direct solar forcing for the terrestrial climate changes. A well-known example is the near-simultaneous occurrence of the Maunder minimum (Eddy 1976) and the Little Ice Age (Shindell et al. 2001). Investigating potential longer term Sun-Earth connections (Sofia \& Li 2001; Bond et al. 2001; Sharma 2002) requires a knowledge of the solar irradiance changes in the past and reliable estimates of irradiance changes in the future. This demands a solid understanding of the physical mechanisms for the solar irradiance cycle.
Following the suggestion by Sofia et al. (1979) that any change in the solar luminosity, $L$, must be accompanied by a change in the radius, $R$, a number of theoretical investigations have attempted to establish the relationship between these changes, denoted as $W=\Delta \ln R / \Delta \ln L$. In each case, the response of a solar model to a particular type of perturbation was calculated, with the perturbation applied at a specific location in the model. From these calculations, it is clear that the derived value of $W$ depends on the form of the perturbation applied to the stellar structure equations and on the location (depth) in the model where the perturbation appears. Two types of perturbations have been investigated extensively, the $\alpha$-perturbations and the $\beta$-perturbations. The $\alpha$-perturbations try to quantify the change in the convective energy flow by assuming that the mixing length parameter $\alpha$ changes (see Ulrich 1975). The $\beta$-perturbations are pressure perturbations caused by magnetic and/or turbulent pressures since we usually denote the ratio of gas pressure to the sum of gas pressure plus non-gas pressure by $\beta$ (see Dearborn \& Blake 1980a, 1980b, 1982, etc.). The usual approach in investigating these perturbations is to use spherically symmetric, hydrostatic stellar evolution codes. The first step is to calibrate the codes by computing a solar model with the mixing length and initial hydrogen/helium abundance required to provide a match to the measured solar radius and luminosity, at the present solar age. Then to apply one or more perturbations and recalibrate the resulting solar model. The zoning and convergence criteria are much tighter than in conventional stellar evolution usage in order to compute the response to the small perturbations being studied. An impulsive, localized perturbation is applied to the model equations for a specified range of zones. The evolution of a perturbed structure (i.e., with the perturbation terms left in place) is then followed over the 
time interval necessary to observe the immediate response and subsequent relaxation. In this approach, all four stellar structure equations play roles and thus four different timescales (dynamic, convective turnover, diffusive, and thermal) are relevant.

Perturbation theories for the irradiance cycle may be illustrative, but physical understanding of solar irradiance cycle based on them are not conclusive for the following reasons:

1. More than one perturbation may play a role at the same time, but the existing calculations include them separately.

2. $\alpha$-perturbations are related to an arbitrary change of the $\alpha$-parameter instead of any physical phenomenon such as magnetic field.

3. The first law of thermodynamics in the presence of magnetic fields is either not explicitly considered or used in incomplete forms in the models.

4. None of the perturbation models attempted to simultaneously explain the observed cycle variations of all global solar parameters and $p$-mode frequencies.

In order to overcome all these shortcomings, a treatment as self-consistent and as systematic as possible of magnetic fields in solar modeling is required. Ultimately, it will be necessary to generalize the one-dimensional stellar structure and evolution code into a three-dimensional stellar structure and evolution code that includes solar turbulence and dynamo by introducing stellar turbulent and magnetic structural variables in addition to the conventional stellar structural variables such as pressure, temperature, radius, and luminosity. Some beginning efforts in this sense can be found in the literature. For example, expansion to twodimensional treatments were carried out by Deupree (1990, 1995, 1998, 2001), and expansion to three dimensions was started by Turcotte et al. (2001). However, these models are not accurate enough to match solar observations, because these authors' objectives did not require high accuracy.

Specific one-dimensional modeling with applications to solar variability studies was initiated by Lydon \& Sofia (1995), updated by Li \& Sofia (2001), and generalized to include turbulence by Li et al. (2002). This paper attempts to use all relevant observations (including oscillations) simultaneously to refine our theoretical understanding of the cycle variations of all solar parameters. We are still using one-dimensional, spherically symmetric models. This approximation is relatively limiting. For example, the only magnetic configuration in one-dimensional codes is shelllike, and here energy flux can only advance to the surface by penetrating the magnetic field shell. If the magnetic field were toroidal, like most dynamo models require, energy flow could circumvent the field.

In order to avoid a perturbation treatment for the magnetic field in stellar modeling, we have to introduce magnetic structural variables in addition to the conventional structure variables such as pressure, temperature, radius, and luminosity. Because magnetic fields are not scalar, Lydon \& Sofia (1995) used two variables, the magnetic energy per unit mass $\chi_{m}$ and the ratio of specific heats due to magnetic fields $\gamma_{m}$ (Endal, Sofia, \& Twigg 1985), to describe magnetic fields in one-dimensional solar models. The governing equations of these two variables are the generation equations for the magnetic fields (for example, the dynamo equations). Since the dynamo equations weakly couple with the other structure equations, Lydon \& Sofia (1995) specify ad hoc $\chi_{m}$ and $\gamma_{m}$ to investigate their influences on the solar models.

Lydon \& Sofia (1995) reformulated the stellar structure equations to ensure conservation of momentum and energy in the presence of magnetic fields. In order to guarantee energy conservation, they use the first law of thermodynamics in the presence of magnetic fields (eq. [14.19] of Callen 1966). Since stellar matter can be considered to be plasma and since plasma has the same permeability as vacuum ( $\mu=\mu_{0}=1$ in Gaussian unit), the equation of state for the magnetic field in plasma is

$$
\boldsymbol{B}=\boldsymbol{H},
$$

where $\boldsymbol{B}$ and $\boldsymbol{H}$ are the local values of induction and field. Consequently, magnetic work takes the form

$$
d W_{m}=d\left(\frac{1}{8 \pi} \int_{V} B^{2} d V\right)=d\left(\frac{B^{2}}{8 \pi \rho}\right) \equiv d \chi_{m}
$$

for a system with unit mass (note: $\rho=1 / V$ in such a system), where $\chi_{m}=B^{2} / 8 \pi \rho$ is the magnetic field energy per unit mass. Therefore, we can write down the first law with a magnetic field as follows:

$$
d U=T d S-P d V+d \chi_{m},
$$

where $U$ is the internal energy of the system, and $P$ is the gas pressure. Lydon \& Sofia (1995) gave the detailed derivation within the framework of the mixing length theory that shows how the convective temperature gradient is modified by a magnetic field when $\chi_{m}$ is considered to be an independent thermodynamic parameter. Recently, Li \& Sofia (2001) showed how this modification changes with both $\chi_{m}$ and $\gamma_{m}\left(\equiv 1+P_{m} / U_{m}\right)$, which are now considered to be independent thermodynamic parameters. The variable $P_{m}=B_{z}^{2} / 8 \pi=\left(\gamma_{m}-1\right) \rho \chi_{m}$ is the magnetic pressure in the vertical direction, and $U_{m}=\chi_{m}$ is the magnetic energy. The total internal energy $U_{\text {tot }}$ per unit mass is the difference between the gas internal energy $U$ and the magnetic energy, $U_{\text {tot }}=U-U_{m}$. From the detailed derivation, we can show that the influence of magnetic fields on the convective transport efficiency of energy results in a modification to the adiabatic gradient (or to the convective velocity within the framework of the mixing length theory), instead of a change of the $\alpha$-parameter as assumed in the $\alpha$-perturbation models (see Lydon \& Sofia 1995 and Li \& Sofia 2001 for the details). Energy conservation is guaranteed in this self-consistent treatment by the use of the first law of thermodynamics in the presence of magnetic fields (i.e., eq. [3]).

Momentum conservation is guaranteed by including the Lorentz force in the hydrostatic equilibrium equation. This effect is equivalent to using the total pressure $P_{\text {tot }}$ to replace the gas pressure in the spherically symmetric hydrostatic equilibrium equation. This is similar to the treatment used in the $\beta$-perturbation models, but the important difference comes from the fact that we use the total pressure as the stellar structure parameter instead of the gas (plus radiation) pressure.

The mass conservation equation is modified indirectly by the equation of state:

$$
\rho=\rho\left(P_{\text {tot }}, T, \chi_{m}, \gamma_{m}\right) .
$$

Consequently, all measurable parameters such as the coefficient of thermal expansion should be redefined at constant 
magnetic field, and new parameters such as density derivatives with respect to the magnetic variables appear (see Lydon \& Sofia 1995 and Li \& Sofia 2001 for details). Because of the length of the magnetic cycle, the generation and destruction of the magnetic fields may be considered to be isothermal. In this case, equation (4) reduces to

$$
\rho=\rho_{0} /\left(1+P_{m} / P_{\text {tot }}\right),
$$

where $\rho$ and $\rho_{0}$ are the density with and without a magnetic field, respectively. This has been checked numerically and found to be a good approximation.

Just as magnetic fields can be described by $\chi_{m}$ and $\gamma_{m}$, turbulence should also be described by the turbulent kinetic energy per unit mass $\chi_{t}$ and the anisotropy parameter $\gamma_{t}$. Robinson et al. (2003) performed a series of large eddy simulations of radiative hydrodynamics in the superadiabatic layers (SALs) of the Sun. Li et al. (2002) define $\chi_{t}$ and $\gamma_{t}$ in terms of the turbulent velocity from the simulations. The difference between the solar model with turbulence and the standard solar model (SSM) is in agreement with the difference between the patched solar model of Rosenthal et al. (1999) and the SSM, measured by using the $p$-mode frequency difference between the models with and without turbulence. In their patched model, the simulated SAL (Stein \& Nordlund 1998) replaces the original SAL of the SSM. This may be considered to be the primary test of the reformulated stellar structure equations with magnetic fields and/or turbulence.

In this paper we include magnetic fields, turbulence, and their interaction in solar models that attempt to reproduce the observed solar cycle-related variations in the global parameters (the total solar irradiance, photospheric temperature, and radius) and the variations in the frequencies of low- and intermediate-degree $p$-mode oscillations. Magnetic fields affect motions of the material in the solar interior by the action of Lorentz forces. When these forces are added to the hydrodynamic equations that govern turbulence in the convection zone of the Sun (Fox, Theobald, \& Sofia 1991; Stein \& Nordlund 2000), they may inhibit turbulence. This is supported by the observation that the shape of granules appears to be different in magnetic regions (Muller 1986; Title et al. 1992), and the measured flow speeds are lower. Since the turbulent pressure is larger than the magnetic pressure, and since magnetic fields tend to inhibit turbulence, the non-gas (turbulent plus magnetic) pressure may decrease with increased activity. This implies an antiphase radius cycle and an in-phase oscillation frequency cycle, in addition to an in-phase photospheric temperature cycle.

The rest of the paper is organized as follows: in $\S 2$ we present the observational constraints against which we test our solar variability models. In $\S 3$ we outline the main ingredients for our solar variability models. The theoretical foundation can be found in our previous publications (Lydon \& Sofia 1995; Li \& Sofia 2001; Li et al. 2002; Sofia \& Li 2001, 2003). The models are described in $\S 4$. In $\S 5$ we compare our models with the observational constraints described in $\S 2$. We present our conclusions in $\S 6$

\section{OBSERVATIONAL CONSTRAINTS}

We list below the specific observations that we expect our models to reproduce.

\subsection{Solar Radiance Variations}

Solar irradiance is known to vary on at least two timescales: the timescale related to solar rotation and that of the solar activity cycle. The amplitude of both these variations is approximately $0.1 \%$. The short-timescale variations are mostly produced by the effect of surface magnetic features (i.e., spots, faculae, and the magnetic network). The cause of the long-timescale variations, which occur in phase with the solar activity cycle (e.g., Fröhlich 2000), is, however, controversial. Many researchers argue that variation on the timescale of the solar cycle is also an effect of the surface features (e.g., Lean et al. 1998). However, many believe that this variation is primarily due to changes in solar luminosity (e.g., Sofia \& Li 2003; Kuhn \& Armstrong 2003). This conclusion is also supported by the observations by Kuhn (1996, 2003; Kuhn et al. 1998), who shows that the faculae, which are supposed to increase irradiance, can actually be dark in the disk center when measured in the continuum wavelengths.

In this work we assume that the changes in solar irradiance are primarily the result of solar luminosity changes, although some fraction of the change is expected to be due to surface features.

\subsection{Variations of Temperature}

The observed solar cycle variation of the photospheric temperature (Gray \& Livingston 1997b) is in phase with solar activity. If the amplitude of the temperature change is indeed what is given by Gray \& Livingston (1997b), then this can account for the entire solar cycle-related variation of the TSI. However, the measurements of Gray \& Livingston (1997a, 1997b), while free from the effects surface magnetic feature, depend on a calibration coefficient that relates the variation of the photospheric temperature to the variation of the depth of the observed spectral lines. They obtained this correlation coefficient empirically from observations of six stars with colors identical to the Sun (Gray \& Livingston 1997a). However, Caccin \& Penza (2002) noted that the gravitational acceleration $g$ for all these stars was not the same, and through theoretical calculations they found a $g$-dependence of the correlation coefficient. This leaves an uncertainty that affects the amplitude of the $11 \mathrm{yr}$ variation of the temperature. Because of this uncertainty, we use only the phase of this change and not the amplitude as the criterion that our models must satisfy.

\subsection{Variations of Solar Radius}

The solar radius is the global property with the most uncertain determination of the solar cycle-related changes. The lack of agreement between near simultaneous groundbased measurements at different locations (often with similar instruments) suggests that atmospheric contamination is so severe that it prevents any meaningful measurement of solar diameter variations at the expected level from the ground. The measurements from space are very few, and some are still being interpreted. The Michelson Doppler Imager (MDI) on board the Solar and Heliospheric Observatory $(\mathrm{SOHO})$ made a few such measurements (Emilio et al. 2000). Because the design of the instrument was not optimized for astrometry, significant corrections had to be applied to the measurements, which introduce some uncertainty in the results. The solar disk sextant (SDS), on the other hand, was specifically designed for this purpose (Sofia, 
Heaps, \& Twigg 1994), and five flights have been carried out over a 9 yr time interval. Although milliarcsecond sensitivity was achieved for individual flights, work is ongoing so that results obtained from different flights can be compared with a similar degree of precision (Egidi et al. 1999; A. Egidi et al. 2003, in preparation; S Sofia et al. 2003, in preparation). Since the SDS has had flights in the Fall of 1992, 1994, 1995, 1996, and 2001, these results will provide a significant constraint for our models. In addition, a French satellite PICARD is under development with the specific purpose of determining the solar radius. Launch is likely to be in 2007 , and the mission is expected to have a lifetime of greater than $3 \mathrm{yr}$. Thus, the reliability of data on radius variations should improve in the near future.

An alternative way of determining variations of the solar radius is by helioseismology, particularly of the $f$-modes of oscillation. Schou et al. (1997) and Antia (1998) have demonstrated that the frequencies of $f$-modes can be used to estimate the solar radius. Since these frequencies have been measured with a precision of a part in $10^{5}$, one may expect to determine the solar radius to similar precision. It must be kept in mind that the radius variations determined by limb observations represent changes at the solar surface, whereas the radius determined from $f$-mode oscillations represents changes at depths from 4 to $10 \mathrm{Mm}$ below the solar surface, so comparisons must be made with care. Although estimates of variations in solar radius from changes in solar $f$-modes are not as confused as direct measurement, nevertheless there is no consensus yet as to the exact amount by which the radius changes. Attempts so far (Dziembowski et al. 1998, 2000, 2001; Antia et al. 2000, 2001) give conflicting results. Dziembowski et al. (1998) using MDI data found that the solar radius reached a minimum around the minimum activity period in 1996 and was larger by about $5 \mathrm{~km}$, 6 months before and after the minimum. But later results using longer time intervals did not find any systematic changes in the solar radius using $f$-mode frequencies (Dziembowski et al. 2000). On the other hand, Antia et al. (2000) using Global Oscillation Network Group (GONG) data found that the solar radius decreased by about $5 \mathrm{~km}$ between 1995 and 1998, and this variation appeared to be correlated with the level of solar activity. Antia et al. (2001) using both GONG and MDI data put an upper limit of 1 $\mathrm{km}$ for the change in solar radius. Dziembowski et al. (2001) claimed a solar radius decrease as a rate of $1.5 \mathrm{~km} \mathrm{yr}^{-1}$ during 1996-2000. Antia et al. (2001) showed that the variation in $f$-mode frequencies is more complex than what had been assumed in earlier studies. These variations can be decomposed into at least two components. One of these components is oscillatory with a period of $1 \mathrm{yr}$; the second is nonoscillatory and probably correlated with solar activity. The oscillatory component is most likely an artifact introduced by the orbital period of the Earth. Antia et al. (2001) also showed that most of the discrepancy between different results about radius variation using $f$-mode frequencies can be explained by the use of data sets that cover different time periods and by the failure to remove the oscillatory component. However, what seems to be clear from the different investigations is that solar radius decreases with solar activity.

Radius determinations from $f$-modes therefore indicate small changes in radius with a phase opposite to that of solar activity. By contrast, direct measurements by MDI (Emilio et al. 2000) indicate equally small changes, but in phase with activity cycle. Early results of the SDS indicate changes in opposite phase with the activity cycle, but substantially larger than the helioseismological ones. Thus, for this study, we only assume that the solar radius varies in antiphase with solar activity.

\subsection{Helioseismic Constraints: Changes in Convection Zone Position}

The position of the base of the convection zone can be determined very precisely by inversions of solar oscillation frequencies (Christensen-Dalsgaard, Gough, \& Thompson 1991; Basu \& Antia 1997). Helioseismic data obtained over the past seven years, however, do not show any observable change of the position of the convection zone base with time (Basu \& Antia 2000; Basu 2002). The errors in the measurements would allow changes of less than a few parts in $10^{-4}$; thus, the constraint we use from these results is that the change of position of the convection zone base in our models must not exceed a few parts in $10^{-4}$.

\subsection{Helioseismic Constraints: Changes in Solar Frequencies}

Solar oscillation frequencies are known to change with changes in solar activity. These observations are perhaps the most sensitive diagnostics of solar models at the present time. In this study, we use $26 \mathrm{MDI}$ data sets containing centroid frequencies determined from measurements made between 1996 May 1 and 2001 April 21. We first sort out the common modes for all data sets, which contain $1381 p$ modes with $6 \leq l \leq 190$, and then take averages for each year. This way we obtain six data sets for 1996-2001. Taking the data set for 1996 (the activity minimum) as the reference set, we obtain frequency changes for each year by subtracting the reference frequencies from that year's data set, as shown in Figure 1.

From Figure 1 we can see that the frequency differences increase with solar activity. The frequency changes are predominantly a function of frequency $\nu$. The degree $(l)$ dependence can be explained as being caused by differences in mode-inertia of the different modes. This suggests that the cause of the frequency changes is restricted to the outer layers of the Sun. In fact, inversions of the frequency differences have revealed that there are no observable structural changes in the inner layers of the Sun (Basu 2002; Basu \& Antia 2002). It must be noted that with the available data sets, one cannot determine the structure of layers with $r>0.97 R_{\odot}$ by inversions.

\section{SOLAR VARIABILITY MODELS}

In order to obtain solar models at different phases of solar activity, we need to reformulate the equations of solar structure by including the effects of magnetic fields. Since magnetic fields affect properties of convection, effects of turbulence also need to be included in the reformulated equations. We describe below how we reformulate the structure equations, how the changes are implemented, and how the presence of magnetic fields changes the radius, effective temperature, and luminosity of the models. See Lydon \& Sofia (1995), Li \& Sofia (2001), Li et al. (2002), and Sofia \& $\mathrm{Li}$ (2003) for the details.

\subsection{Reformulation of Stellar Structure Equations}

The stellar structure equations approximately take on the same form as for the standard stellar structure equations 


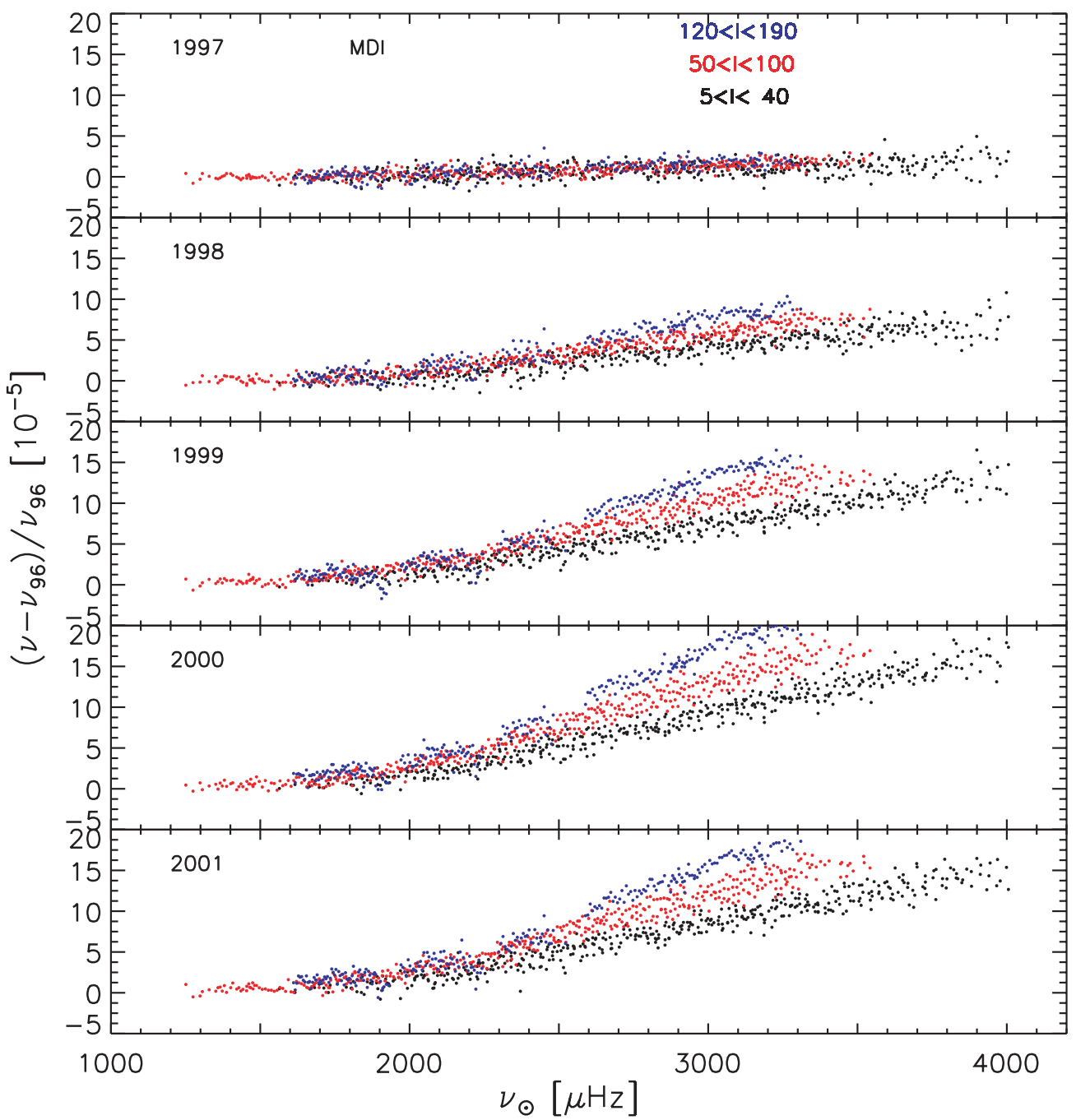

FIG. 1.-Observed relative $p$-mode frequency variations by MDI as functions of frequency and angular degree

when a magnetic field and/or turbulence is included:

$$
\begin{gathered}
\partial \log P / \partial x=-G M^{2} / 4 \pi P r^{4}, \\
\partial \log T / \partial x=\nabla(\partial \log P / \partial x), \\
\partial \log r / \partial x=M / 4 \pi \rho r^{3}, \\
\partial L / \partial x=\ln 10\left(M / L_{\odot}\right)(\epsilon-T d S / d t),
\end{gathered}
$$

where $x=\log M$ is chosen to be the independent variable; $\nabla=\nabla_{c}$ (to be defined below) in the convection zone, while $\nabla=\nabla_{\text {rad }}$ in the radiation zone, where $\nabla_{\text {rad }}$ is the radiative gradient. The neglected terms are 1 order of magnitude smaller than the modification to the equation of state. All units are in cgs, except for the luminosity $(L)$, which is in solar units.

The magnetic and turbulent influences are included by the following:

1. Using equation (3), the first law of thermodynamics in the presence of magnetic fields or turbulence.

2. Using $\chi$ and $\gamma$ as new stellar structure variables, in addition to the conventional stellar structure variables, $P_{0}$, $T, r$, and $L$
3. Using the total pressure

$$
P=P_{0}+(\gamma-1) \chi \rho
$$

as the stellar structure variable, where $\chi$ and $\gamma$ are considered to be thermodynamic variables, $P_{0}$ is the gas pressure.

4. Using the mixing length approximation to calculate influences of magnetic fields and/or turbulence on the temperature gradient in the convection zone. The result is

$$
\nabla_{c}=\nabla_{\mathrm{ad}}^{\prime}+\left(y / V \gamma_{o}^{2} C\right)(1+y / V) .
$$

The quantities $\gamma_{0}, C, V$, and $y$ are defined by

$$
\begin{gathered}
\gamma_{0}=\left[\left(c_{p} \rho\right) /\left(2 a c T^{3}\right)\right]\left[1+(1 / 3) \omega^{2}\right] / \omega, \\
C=\left(g / l_{m}^{2} \delta\right) / 8 H_{p}, \\
\nabla_{\mathrm{ad}}^{\prime}=\left\{1-f\left[(\nu / \mu) \nabla_{\chi}+\left(\nu^{\prime} / \mu\right) \nabla_{\lambda}\right]\right\} \nabla_{\mathrm{ad}}, \\
V=1 /\left[\gamma_{0} C^{1 / 2}\left(\nabla_{\mathrm{rad}}-\nabla_{\mathrm{ad}}^{\prime}\right)^{1 / 2}\right], \\
A=(9 / 8)\left[\omega^{2} /\left(3+\omega^{2}\right)\right], \\
y=v_{\text {conv }} \gamma_{o} V .
\end{gathered}
$$

Here $\omega=\kappa \rho l_{m}, g$ is the gravity acceleration, $H_{p}$ is the 
pressure scale height, $l_{m}$ is the mixing length, $\nabla_{\text {rad }}$ is the radiative temperature gradient, $f$ is a dimensionless parameter that determines the influence of magnetic field on radiative loss of a convective element, and $\mu$ is the compressibility coefficient at a constant temperature and a constant magnetic field. The derivatives relevant to the magnetic field and turbulence are

$$
\begin{gathered}
\nu=-(\partial \ln \rho / \partial \ln \chi)_{T, P, \gamma}, \nabla_{\chi}=\partial \ln \chi / \partial \ln P, \\
\nu^{\prime}=-(\partial \ln \rho / \partial \ln \gamma)_{T, P, \chi}, \quad \nabla_{\gamma}=\partial \ln \gamma / \partial \ln P .
\end{gathered}
$$

From these reformulated stellar structure equations, we can see the following:

1. The magnetic fields and/or turbulence do not directly change the $\alpha$ parameter but directly change the convective velocity $v_{\text {conv }}$ and the convective temperature gradient $\nabla_{c}$. Nevertheless, we may fine-tune $\alpha$ to mimic these effects (hereafter we use the term "effect" to replace "perturbation" since we do not use any perturbation treatment).

2. When we use the total pressure as the stellar structure parameter, we automatically include the $\beta$-effects. However, the pressure or $\beta$ effects are much smaller than the $\alpha$-effects caused by the magnetic or turbulent energy associated with the pressure effects, as verified by Li et al. (2002) for turbulence.

\subsection{Implementation}

\subsubsection{Inclusion of Magnetic Fields}

To follow the behavior of the solar model in response to a variable magnetic field $\boldsymbol{B}=\left(B_{t}, B_{p}\right)$ in its interior, we define the magnetic energy per unit mass, $\chi$, and the effective ratio of specific heats due to the magnetic perturbations, $\gamma$ as follows:

$$
\begin{gathered}
\chi=\left(B^{2} / 8 \pi\right) / \rho, \\
\gamma=1+2 B_{t}^{2} / B^{2},
\end{gathered}
$$

where $B^{2}=B_{t}^{2}+B_{p}^{2}, B_{t}$ is the horizontal component of the magnetic field, $B_{p}$ the radial component. The former describes the magnetic perturbation strength, and the latter describes the tensor feature of the magnetic pressure. In general, the determination of $\chi$ and $\gamma$ requires a comprehensive understanding of the dynamics of turbulence in the solar convection zone, an undertaking that is impractical at present. Therefore, we specify $\chi$ as functions of time $t$ (or sunspot number $R_{Z}$ ) and the mass depth $M_{D}=$ $\log \left(1-M / M_{\odot}\right)$ as

$\chi\left(M_{D}, R_{Z}\right)=\chi_{0}\left(R_{Z}\right) \exp \left[-\frac{1}{2}\left(M_{D}-M_{D c}\right)^{2} / \sigma^{2}\right]$,

where $M_{D c}$ specifies the location and $\sigma$ specifies its width. $\chi_{0}$ is determined by

$$
\chi_{0}\left(R_{Z}\right)=\frac{B_{0}^{2}}{8 \pi \rho_{c}}\left\{140+\left[1+\log \left(1+R_{Z}\right)\right]^{5}\right\}^{2},
$$

where $B_{0}$ is an adjustable parameter (unit: Gauss), and $\rho_{c}$ is the density at the mass depth of $M_{D c}$. We use $\gamma=2$, which maximizes the magnetic effects.

In this case the magnetic variable-related derivatives reduce to

$$
\nu=\chi \rho / P_{T}, \nu^{\prime}=2 \nu, \nabla_{\gamma}=0
$$

$$
\begin{aligned}
\nabla_{\chi} & =\frac{\partial \ln \chi}{\partial \ln M_{D}} \frac{\partial \ln M_{D}}{\partial \ln M} \frac{\partial \ln M}{\partial \ln P_{T}} \\
& =-\frac{M_{D}\left(M_{D}-M_{D c}\right)}{\sigma^{2} \ln 10} \frac{1-10^{M_{D}}}{10^{M_{D}}} \frac{4 \pi P_{T} r^{4}}{G M_{\odot}^{2}\left(1-10^{M_{D}}\right)^{2}} .
\end{aligned}
$$

\subsubsection{Inclusion of Turbulence}

Turbulence is described by turbulent velocities. Turbulent velocities are defined by the velocity variance:

$$
v_{i}^{\prime \prime}=\left(\overline{v_{i}^{2}}-{\overline{v_{i}}}^{2}\right)^{1 / 2},
$$

where the overbar denotes a combined horizontal and temporal average, and $v_{i}$ is the total velocity, obtained by realistic three-dimensional solar convection simulations. Using the turbulent velocity, the turbulent kinetic energy per unit mass, $\chi$, and the effective ratio of specific heats due to turbulence, $\gamma$, are defined to be

$$
\begin{gathered}
\chi=\frac{1}{2}\left(v^{\prime \prime}\right)^{2}, \\
\gamma=1+2\left(v_{z}^{\prime \prime} / v^{\prime \prime}\right)^{2},
\end{gathered}
$$

where $v^{\prime \prime 2}=v_{x}^{\prime \prime 2}+v_{y}^{\prime \prime 2}+v_{z}^{\prime \prime 2}$. When turbulence is isotropic $\left(v_{z}^{\prime \prime}=v_{x}^{\prime \prime}=v_{y}^{\prime \prime}\right), \gamma=5 / 3$; when turbulence is completely anisotropic $\left(v_{z}^{\prime \prime}=v^{\prime \prime}\right.$ or $\left.v_{z}^{\prime \prime}=0\right), \gamma=3$ or 1 , respectively. See Li et al. (2002) for the details.

To obtain reliable turbulent velocities, we have performed large eddy simulations of three-dimensional radiative hydrodynamics in the SAL of the Sun (Robinson et al. 2003) by solving the full set of governing equations.

The turbulent velocities as obtained by the simulations are shown in Figure 2 for the present Sun. Using these we can define $\chi$ and $\gamma$, as shown in Figures 3 and 4. In order to compare our results with similar simulations by the other authors (Stein \& Nordlund 1998; Asplund et al. 2000), we show our turbulent pressure $P_{\text {turb }}=(\gamma-1) \chi \rho$ in Figure 5 . Our maximum turbulent pressure is slightly smaller than theirs. The reason is likely to be that our vertical resolution is poorer than theirs (see Robinson et al. 2003 for details). Note that our spatial grid scale is $114 \times 114 \times 170$ for a

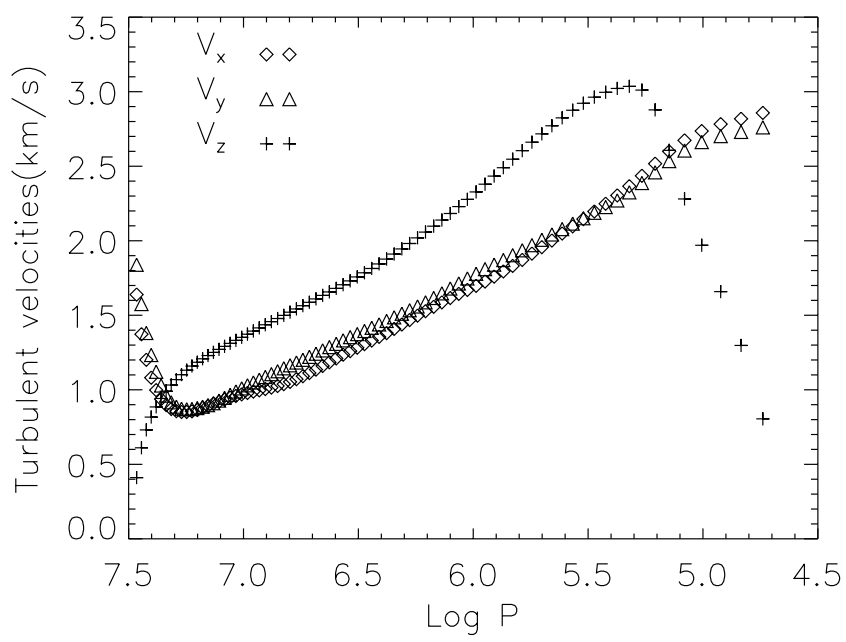

FIG. 2.-Turbulent velocities in the horizontal $\left(v_{x}\right.$ and $\left.v_{y}\right)$ and vertical $\left(v_{z}\right)$ directions vs. depth. The closeness of the two horizontal velocities confirms that the simulation is near statistical convergence. 


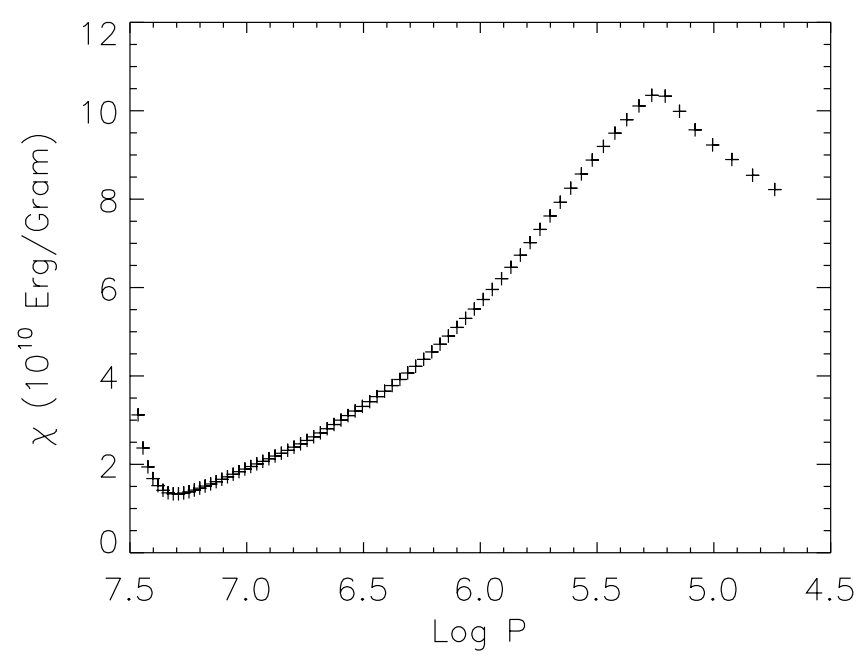

FIG. 3.-Turbulent kinetic energy per unit mass as a function of depth for the Sun.

box of $5.4 \times 5.4 \times 2.8 \mathrm{Mm}$, while their best scale is $253 \times 253 \times 163$ for a box of $6 \times 6 \times 3 \mathrm{Mm}$.

In this case $\nabla_{\chi}$ and $\nabla_{\gamma}$ can be obtained by performing the numerical derivation of $\chi$ and $\gamma$ with respect to pressure.

\subsubsection{Inclusion of Both Turbulence and Magnetic Fields}

There is evidence from observations and numerical experiments that the solar magnetic field consists of two components. One is the small-scale photospheric field, which is believed to result from local dynamo action in the convective flows at or near the solar surface (e.g., Cattaneo 1999), independent of solar rotation. Another is the largescale (or global) solar magnetic field, which is likely to be generated deeper within the Sun, probably at the base of the convection zone (e.g., Tobias et al. 2001). The small-scale field is present even in the quiet photosphere (and at the activity minimum).

According to the scenario above, the large-scale magnetic field in the SAL of the Sun can be considered to be an exter-

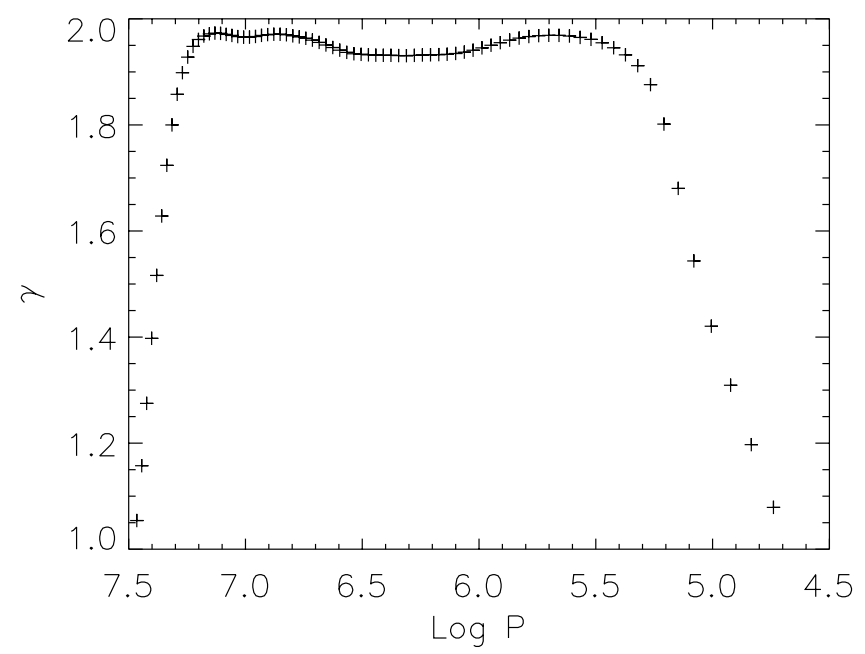

FIG. 4.-Specific heat ratio due to turbulence as a function of depth in the Sun.

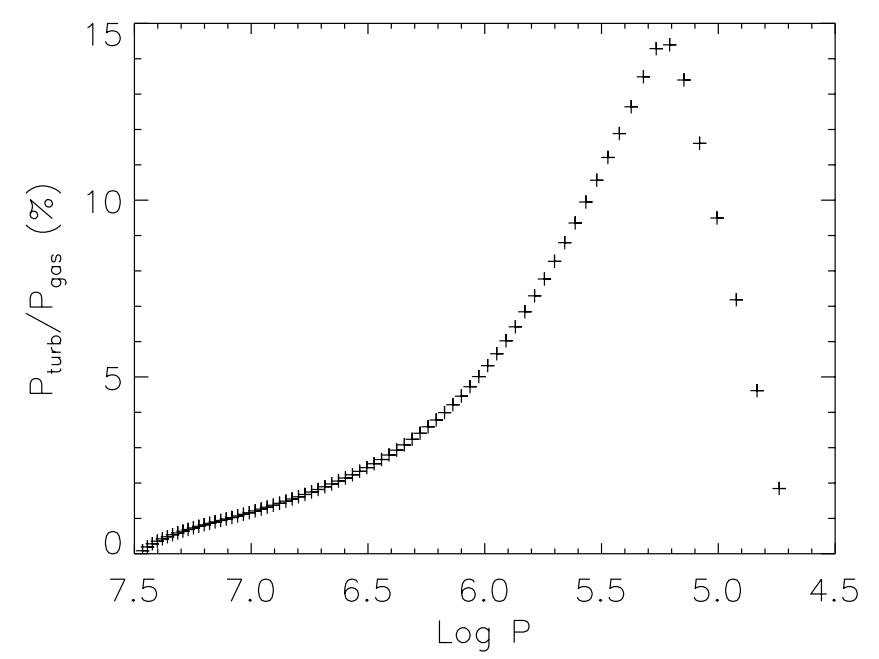

FIG. 5.- Ratio of turbulent to total pressure in the outer layers as a function of depth in the Sun.

nally applied field. Such an external magnetic field tends to suppress turbulence. By defining the plasma $\beta$ to be the ratio of the magnetic pressure to the gas pressure, one might plausibly assume that the larger the plasma $\beta$, the stronger the magnetic suppression effect of turbulence. Therefore, we can use the sunspot number $R_{Z}$ to model the modulation factor

$$
s\left(R_{Z}\right)=\left(R_{Z} / 157\right)^{0.4}
$$

Using the modulation factor as a temporal factor, and designating $\chi$ obtained in the previous subsection $(\S 3.2 .2)$ as $\chi_{0}$, we model the magnetically modulated turbulence as

$$
\chi_{t}= \begin{cases}\left(1-f_{1} s\right) \chi_{0} & \log P \leq f_{3}, \\ \chi_{0} & \log P>f_{3},\end{cases}
$$

where $f_{1}$ is a parameter that models the modulation depth, and $f_{3}$ is a parameter that models the depth dependence of the effect of magnetic modulation on turbulence. We assume that the magnetic energy per unit mass, $\chi_{m}$, is proportional to the variable component of $\chi_{t}$,

$$
\chi_{m}=f_{2} s \chi_{0},
$$

where $f_{2}$ is a parameter that reflects the generation efficiency of magnetic fields by turbulence. Consequently, the total nonthermal energy per unit mass when magnetically modulated turbulence and magnetic fields are present is obtained by summing them up:

$$
\chi= \begin{cases}{\left[1-\left(f_{1}-f_{2}\right) s\right] \chi_{0}} & \log P \leq f_{3} \\ \left(1+f_{2} s\right) \chi_{0} & \log P>f_{3}\end{cases}
$$

Note that the temporal changes of $\chi_{m}, \chi_{t}$, and $\chi$ are modeled by $s=s\left(R_{Z}\right)$ defined in equation (19).

Similarly, we define $\gamma_{m}=\gamma_{t}=\gamma_{0}$, where $\gamma_{0}$ is given by Figure 4. The total $\gamma$ is defined as follows:

$$
\gamma=\left(\gamma_{m} \chi_{m}+\gamma_{t} \chi_{t}\right) / \chi
$$

Obviously, $\gamma=\gamma_{t}\left(\right.$ or $\left.\gamma_{m}\right)$ when $\chi_{m}=0\left(\right.$ or $\left.\chi_{t}=0\right)$. 
In this case we can use the definitions of $\chi$ and $\gamma$ to derive the expressions for $\nabla_{\chi}$ and $\nabla_{\gamma}$ by $\nabla_{\chi_{m}}$, etc., as follows:

$$
\begin{gathered}
\nabla_{\chi}=\frac{\chi_{m}}{\chi} \nabla_{\chi_{m}}+\frac{\chi_{t}}{\chi} \nabla_{\chi_{t}}, \\
\nabla_{\gamma}=\frac{\chi_{m} \gamma_{m}\left(\nabla_{\gamma_{m}}+\nabla_{\chi_{m}}\right)+\chi_{t} \gamma_{t}\left(\nabla_{\gamma_{t}}+\nabla_{\chi_{t}}\right)}{\gamma \chi}-\nabla_{\chi} .
\end{gathered}
$$

We therefore have four free parameters for turbulence, $f$, $f_{1}, f_{2}$, and $f_{3}$. Not all models have all four free parameters. We try to determine these parameters by trying to reproduce the observed variability constraints. Although we treat these quantities as free parameters, each of these has a physical meaning and is therefore derivable from first principles. Detailed magneto-hydrodynamic simulations, which are not yet available, should give us the values of the parameters $f, f_{1}, f_{2}$, and $f_{3}$.

\subsection{Effect of Magnetic Fields on Radius}

Solar radius change is mostly caused by the $\beta$-effects. This is confirmed by our numerical experiments. Using equation (5), we can estimate the radius variations owing to the nongas pressure $P_{N}$ by integrating the mass conservation equation with and without non-gas pressure, and then taking the difference between them:

$$
\Delta R \approx \int_{0}^{M_{\odot}} \frac{P_{N} d M}{4 \pi r^{2} \rho_{0} P_{T}} .
$$

From this formula it can be seen that the magnetic pressure always increases the solar radius, provided that there is a toroidal component of the magnetic field. The resonant cavity of the $p$-mode eigenfrequencies increases when the radius increases. The $p$-mode eigenfrequencies decrease when the resonant cavity increases. Consequently, an in-phase radius cycle implies an antiphase $p$-mode frequency cycle, and vice versa. Therefore, in order to obtain the observed in-phase $p$ mode frequency cycle, we have to include both magnetic fields and turbulence and assume that the turbulent pressure is larger than the magnetic pressure in the solar interior. In this case the total non-gas pressure $P_{N}$ equals the sum of the magnetic pressure $P_{m}=f_{2} s(\gamma-1) \rho \chi_{0}$ and the turbulent pressure $\left(1-f_{1} s\right)(\gamma-1) \rho \chi_{0}$ :

$$
P_{N}=\left[1-\left(f_{1}-f_{2}\right) s\right](\gamma-1) \rho \chi_{0} .
$$

From equation (27) it can be seen that $P_{\chi}$ will decrease with increase in activity if $f_{1}>f_{2}$. In this case we can see from equation (26) that we can get an antiphase radius cycle.

\subsection{Effect on Effective Temperature}

Magnetic fields and turbulence affect the temperature gradient in the convection zone. This effect can be implemented by using an effective adiabatic temperature gradient (see eq. [11]). The change of the temperature gradient can be estimated by the difference between the effective and actual adiabatic gradients:

$$
\Delta \nabla \approx-f\left(\nu \nabla_{\chi}+\nu^{\prime} \nabla_{\gamma}\right) \nabla_{\mathrm{ad}} / \mu,
$$

where $\nabla=\partial \ln T / \partial \ln P$ is the actual temperature gradient. The relative variation of the surface (or effective) temperature can be estimated by using a similar argument as in equation (26)

$$
\Delta \ln T_{\mathrm{eff}} \approx-\int_{0}^{M_{\odot}} \frac{G M}{4 \pi r^{4} P}(\Delta \nabla) d M .
$$

\subsection{Effect on Luminosity}

The luminosity at the surface is determined by both the radius $R$ and the effective temperature $T_{\text {eff }}$ :

$$
L=4 \pi R^{2} \sigma T_{\mathrm{eff}}^{4},
$$

where $\sigma$ is the Stefan constant. Therefore, the observed luminosity variation can be estimated by

$$
\Delta \ln L=2 \Delta \ln R+4 \Delta \ln T_{\text {eff }} .
$$

Numerical models presented below give $\Delta \ln L, \Delta R$, and $\Delta T_{\text {eff }}$ at the same time. Therefore, equation (30) is used to check if the results are self-consistent.

\section{THE MODELS}

We use the latest version of the Yale Rotating Evolution Code (YREC) which incorporates the OPAL equation of state (Rogers, Swenson, \& Iglesias 1996), OPAL opacities (Iglesias \& Rogers 1996), low-temperature opacities (Alexander \& Ferguson 1994), and heavy-element diffusion (Guenther \& Demarque 1997). The model atmosphere is constructed using the empirical Krishna-Swamy (KS; 1966) $T-\tau$ relation. This version is well tested by Winnick et al. (2002). We modify this code to include both magnetic fields and turbulence as described above.

We have two kinds of models:

1. Models for which the magnetic field has a Gaussian profile. For these models parameters $f_{2}$ and $f_{3}$ are irrelevant. These models are summarized in Table 1.

2. Models for which the magnetic energy density is assumed to be proportional to the turbulent kinetic energy density. These models could have all four free parameters, $f$, $f_{1}, f_{2}$, and $f_{3}$, for turbulence. These models are summarized in Table 2 .

Models in category 1 can be further subdivided into three groups, I, II, and III, described below. Models in category 2 can be subdivided into four groups I-IV described below. These subdivisions are as follows:

1. Group I.-Models with no effect of turbulence (models A-F from category 1 , and model $\mathrm{M}$ from category 2 ). This automatically implies that $f=1$ and the other parameters that describe turbulence $\left(f_{1}, f_{2}\right.$, and $\left.f_{3}\right)$ are irrelevant. Another freedom we have in these models is the position and amplitude of the magnetic field. The amplitude of the field for a given position is determined by a fit to the amplitude of the observed luminosity variations.

2. Group II.--Models with partial effect of turbulence (models $\mathrm{G}-\mathrm{J}$ from category 1 , and model $\mathrm{N}$ from category 2 ), in which the radiative loss of a convective eddy is assumed to be affected by turbulence, but neither turbulent kinetic energy nor turbulent pressure is included. This effect is modeled by parameter $f$, which is determined by a fit to the amplitude of the observed photospheric temperature variations. We find $f=3 ; f_{1}$ and $f_{3}$ are again irrelevant. The parameter $f_{2}$ is irrelevant for models $\mathbf{G}-\mathbf{J}$, while $f_{2}$ is deter- 
TABLE 1

Solar Variability Models with a Gaussian $\boldsymbol{B}$ Profile

\begin{tabular}{|c|c|c|c|c|c|c|c|c|c|c|c|c|c|}
\hline $\begin{array}{l}\text { Group } \\
\text { (1) }\end{array}$ & $\begin{array}{l}\text { Model } \\
\text { (2) }\end{array}$ & $\begin{array}{l}f^{\mathrm{a}} \\
(3)\end{array}$ & $\begin{array}{l}f_{1}^{\mathrm{b}} \\
(4)\end{array}$ & $\begin{array}{l}M_{D c} \\
(5)\end{array}$ & $\begin{array}{c}\sigma \\
(6)\end{array}$ & $\begin{array}{c}B_{0} \\
(\mathrm{G}) \\
(7)\end{array}$ & $\begin{array}{c}\log P \\
(8)\end{array}$ & $\begin{array}{c}\text { Depth } \\
(\mathrm{Mm}) \\
(9)\end{array}$ & $\begin{array}{c}\Delta \ln L \\
(\%) \\
(10)\end{array}$ & $\begin{array}{c}\Delta \ln R \\
\left(10^{-6}\right) \\
(11)\end{array}$ & $\begin{array}{l}\Delta T \\
(\mathrm{~K}) \\
(12)\end{array}$ & $\begin{array}{c}\Delta R_{\mathrm{CZ}} \\
\left(R_{\odot}\right) \\
(13)\end{array}$ & $\begin{array}{c}\Delta B \\
(\mathrm{kG}) \\
(14)\end{array}$ \\
\hline \multirow{3}{*}{ I . . } & B & 1 & $\ldots$ & -2 & 0.2 & 2800 & 13.22 & 138.69 & 0.061 & 350 & -0.13 & 0.067 & 690 \\
\hline & $\mathrm{C}$ & 1 & $\ldots$ & -4.25 & 0.2 & 200 & 10.74 & 20.07 & 0.074 & 47 & 0.94 & 0 & 49 \\
\hline & $\mathrm{D}$ & 1 & $\ldots$ & -6.5 & 0.2 & 17 & 8.46 & 4.45 & 0.078 & 10 & 1.09 & 0 & 4.2 \\
\hline \multirow[t]{4}{*}{ II } & G & 3 & $\ldots$ & -4.25 & 0.2 & 120 & 10.74 & 20.07 & 0.074 & 16 & 1.02 & 0 & 30 \\
\hline & $\mathrm{H}$ & 3 & $\ldots$ & -6.5 & 0.2 & 10 & 8.46 & 4.45 & 0.080 & 3.2 & 1.15 & 0 & 2.5 \\
\hline & I & 3 & $\ldots$ & -7.5 & 0.2 & 3.8 & 7.46 & 2.43 & 0.074 & 2.0 & 1.06 & 0 & 1.4 \\
\hline & $\mathrm{J}$ & 3 & $\ldots$ & -9.5 & 0.2 & 0.55 & 5.53 & 0.38 & -0.083 & 0.45 & -1.20 & 0 & 0.15 \\
\hline \multirow[t]{2}{*}{ III } & $\mathrm{K}$ & 1 & 0 & -9.5 & 0.2 & 0.7 & 5.53 & 0.38 & -0.109 & 2.2 & -1.59 & 0 & 0.17 \\
\hline & $\mathrm{L}$ & 3 & 0 & -9.5 & 0.2 & 0.6 & 5.53 & 0.38 & -0.109 & -0.04 & -1.46 & 0 & 0.15 \\
\hline
\end{tabular}

NotE.-See $\S 4$ for definition of the terms used in the heading of the table.

a $f=1$ means that turbulence does not affect the radiation loss of a flow eddy in the convection zone as in Lydon \& Sofia 1995, $f=3$ implies that turbulence inhibits the radiation loss of a flow eddy as in Li \& Sofia 2001.

${ }^{\mathrm{b}}$ Ellipses indicate that the parameter is irrelevant; 0 means that steady turbulent pressure and turbulent kinetic energy are included in the way described in Li et al. 2002.

mined by a fit to the amplitude of the observed luminosity variations for model $\mathrm{N}$. We have another free parameter for models $\mathbf{G}-\mathbf{J}$, the amplitude of the field for a given location of the magnetic field. As in case I above, the amplitude is determined by a fit to the amplitude of the observed luminosity variations.

3. Group III.-Fully turbulent models (models $\mathrm{K}$ and L from category 1 , and models $\mathrm{O}$ and $\mathrm{P}$ from category 2 ), in which both turbulent pressure and turbulent kinetic energy are included. Models L and $\mathrm{P}$ assume extra radiative loss for a convective eddy by turbulence $(f=3)$, while models $\mathrm{K}$ and $\mathrm{O}$ do not assume extra radiative loss $(f=1)$. In these models, turbulence is assumed not to vary with time, which implies that $f_{1}=0$. The parameter $f_{3}$ is irrelevant. $f_{2}$ is irrelevant for models $\mathrm{K}$ and $\mathrm{L}$ but determined by a fit to the amplitude of the observed luminosity variations for models $\mathrm{O}$ and $\mathrm{P}$. The amplitude of the field for a given location of the magnetic field for models $\mathrm{K}$ and $\mathrm{L}$ is determined by a fit to the amplitude of the observed luminosity variations.

4. Group IV.-Magnetically modulated turbulent models (models Q and R), in which time-varying turbulent pressure and time-varying turbulent kinetic energy are modeled by parameters $f_{1}$ and $f_{3}$, and the time-varying magnetic field is modeled by $f_{2}$. When the value of $f$ is specified ( 1 or 3 ), we determine $f_{1}$ and $f_{2}$ by a fit to the amplitude of the observed luminosity and photospheric temperature variations. Parameter $f_{3}$ is then determined by a fit to the observed $p$-mode frequency variations.

Figures 6 and 7 show the differences of magnetic fields between the solar activity maximum and minimum for models A-J (Gaussian profile) and models M-R (turbulent profile). The magnetic field profile for models $\mathrm{K}-\mathrm{L}$ is similar to model F or model $\mathrm{J}$.

In both Tables 1 and 2, column (1) marks the group, column (2) names the model, column (3) shows the parameter $f$ to indicate if turbulence affects the radiation loss of a convective eddy ( 1 , no; 3 , some), and column (4) indicates whether the turbulent kinetic energy (and turbulent pressure) is included or not (ellipses denote that they are not included).

In Table 1, column (5) lists $M_{D c}$ that is the depth of the peak of the applied magnetic field, column (6) lists $\sigma$-the width of the Gaussian profile, and column (7) lists $B_{0}-$ proportional to the amplitude of the field. Columns (8) and (9) express $M_{D c}$ in terms of the pressure variable (used in

TABLE 2

Solar Variability Models with a $\boldsymbol{B}$ Profile from Turbulence

\begin{tabular}{|c|c|c|c|c|c|c|c|c|c|c|}
\hline $\begin{array}{l}\text { Group } \\
\text { (1) }\end{array}$ & $\begin{array}{l}\text { Model } \\
\text { (2) }\end{array}$ & $\begin{array}{c}f \\
(3)\end{array}$ & $\begin{array}{l}f_{1}{ }^{\mathrm{a}} \\
(4)\end{array}$ & $\begin{array}{l}f_{2} \\
(5)\end{array}$ & $\begin{array}{l}f_{3} \\
(6)\end{array}$ & $\begin{array}{c}\Delta \ln L \\
(\%) \\
(7)\end{array}$ & $\begin{array}{c}\Delta \ln R \\
\left(10^{-6}\right) \\
\quad(8)\end{array}$ & $\begin{array}{l}\Delta T \\
(\mathrm{~K}) \\
(9)\end{array}$ & $\begin{array}{c}\Delta R_{\mathrm{CZ}} \\
\left(R_{\odot}\right) \\
(10)\end{array}$ & $\begin{array}{c}\Delta B \\
(\mathrm{kG}) \\
(11)\end{array}$ \\
\hline I & M & 1 & $\ldots$ & 0.04 & $\ldots$ & -0.105 & 2.5 & -1.62 & 0 & 0.27 \\
\hline 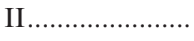 & $\mathrm{N}$ & 3 & $\ldots$ & 0.022 & $\ldots$ & -0.105 & 0.7 & -1.52 & 0 & 0.20 \\
\hline \multirow[t]{2}{*}{ III …................... } & $\mathrm{O}$ & 1 & 0 & 0.036 & $\ldots$ & -0.099 & 0.2 & -1.42 & 0 & 0.26 \\
\hline & $P$ & 3 & 0 & 0.11 & $\ldots$ & -0.099 & 0.6 & -1.33 & 0 & 0.45 \\
\hline \multirow[t]{2}{*}{ 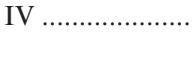 } & Q & 3 & 0.155 & 0.08 & 6.4 & -0.066 & -5.0 & -0.94 & 0 & 0.38 \\
\hline & $\mathrm{R}$ & 1 & 0.155 & 0.08 & 6.4 & 0.106 & -3.7 & 1.54 & 0 & 0.38 \\
\hline
\end{tabular}

NoTE.-See $\S 4$ for definition of the terms used in the heading of the table.

${ }^{a}$ Ellipses indicate that the parameter is irrelevant; 0 means that steady turbulent pressure and turbulent kinetic energy are included in the way described in $\mathrm{Li}$ et al. 2002, and a nonzero $f_{1}$ means that a magnetically modulated (varying) turbulent kinetic energy and turbulent pressure are included. 


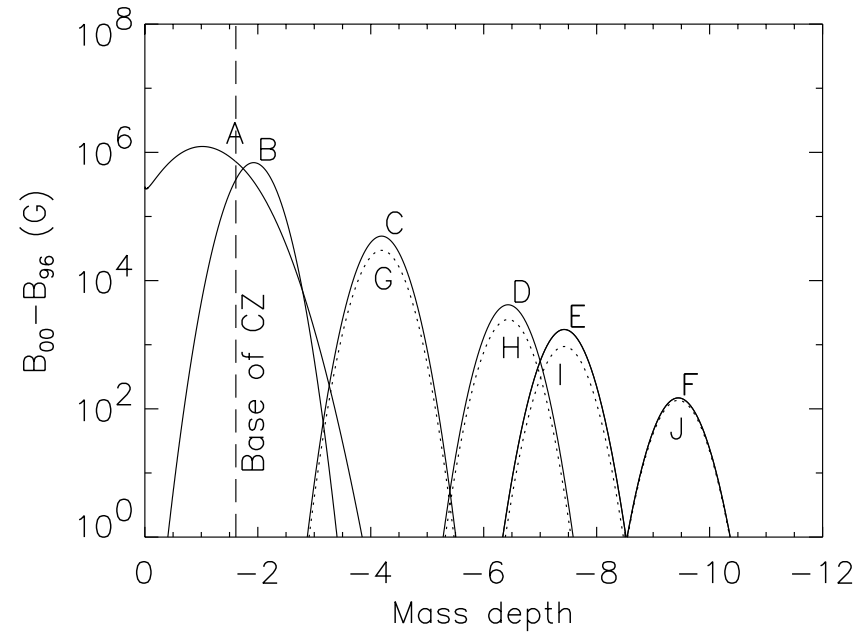

FIG. 6.-Differences of magnetic fields at the solar activity maximum and minimum as functions of mass depth for models $\mathrm{A}-\mathrm{F}$.

turbulence simulations) and physical depth, respectively. Columns (10)-(12), respectively, list the variations of global solar parameters $L, T_{\text {eff }}$, and $R$ between the years 2000 and 1996. The last two columns give the corresponding variations for the convective depth and the maximal values of the magnetic fields. Roughly speaking, when turbulence is taken into account partially (as in models $\mathrm{G}-\mathbf{J}$ ), the required magnetic field strength to produce the observed irradiance cycle variations decreases, and so does the contribution from the radius change to the irradiance change. We assume that the observed irradiance variation is proportional to the luminosity variation.

In Table 2, column (5) lists $f_{2}$, which specifies the ratio of the magnetic energy over the turbulent kinetic energy. Column (6) lists $f_{3}$, which indicates the depth dependence of the magnetic modulation of turbulence. Columns (7)-(11) are the same as columns (10)-(14) of Table 1.

In order to obtain models A-J (and M, N), we first obtain a standard solar model by evolving from the zero-age main sequence to the current solar age (4.5 Gy) with $\left(X_{\text {init }}, Z_{\text {init }}, \alpha\right)=(0.6957,0.022,2.0982)$, where $\alpha$ is the

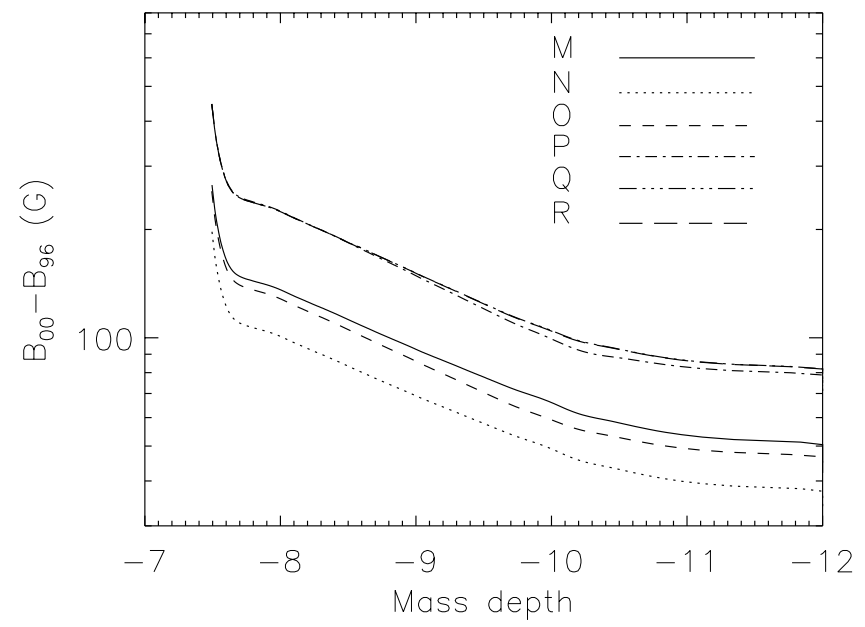

FIG. 7.-Differences of magnetic fields at the solar activity maximum and minimum as functions of mass depth for models $\mathrm{M}-\mathrm{R}$. usual mixing length parameter. This is the best SSM one can get with YREC (Winnick et al. 2002). Then we turn on the varying magnetic field as defined above and reduce the time step to 1 yr to follow the cycle variations of the Sun. In order to obtain Models $\mathrm{K}-\mathrm{L}$ (and $\mathrm{O}-\mathrm{R}$ ) we first obtain a solar model by including the turbulent kinetic energy and turbulent pressure from the three-dimensional numerical simulation and by evolving from the zero-age main sequence to the current solar age $(4.5 \mathrm{~Gy})$ with $\left(X_{\text {init }}, Z_{\text {init }}, \alpha\right)=$ (0.6957, 0.022, 2.2468), as done by Li et al. (2002). This model is called a turbulent solar model. Then we turn on a varying magnetic field and reduce the time step to $1 \mathrm{yr}$ to follow the cycle variations of the Sun.

\section{TESTS OF MODELS}

\subsection{Tests of Global Parameter Variations}

All the models described above are constructed to produce a cycle luminosity variation of approximately onetenth of one percent. However, we find that while the luminosity changes of models $\mathrm{A}-\mathrm{E}, \mathrm{G}-\mathrm{I}$, and $\mathrm{R}$ are in phase with solar activity, those of models $\mathrm{F}$ and $\mathrm{J}-\mathrm{Q}$ have an opposite phase. Therefore, under our assumption that the irradiance variation is directly proportional to the luminosity variation only models A-E, G-I, and R can pass this test.

In addition, our models were required to show a photospheric temperature variation in phase with activity. Only models C-E, G-I, and R satisfy this constraint (as can be seen from Tables 1 and 2). Similarly, our models are required to have radius variations in antiphase with solar activity. This antiphase behavior of the solar radius is only seen in models L, Q, and R.

\subsection{Helioseismic Tests \\ 5.2.1. CZ base}

As can be seen from Tables 1 and 2, the position of the $\mathrm{CZ}$ base changes substantially for models $\mathrm{A}$ and $\mathrm{B}$. The position of the CZ base remains constant for all other models. Therefore, while this test allows us to rule out models A and $\mathrm{B}$, it does not discriminate between the other models.

\subsubsection{Frequency Changes in the Models}

We can compare the observed solar cycle-related frequency changes with those obtained for our models. We use the pulsation code of Guenther (1994) to calculate $p$-mode frequencies of our models. We also look at time-variations of the position of the base of the convection zone. The results are described below:

1. Nonturbulent models (models $A-F$ and $M$ ).--Models A and $\mathrm{B}$ are unacceptable because of the change they show in the position of the base of the convection zone. Our conclusion is strengthened by the frequency variations of these models. Figure 8 shows the calculated relative $p$-mode frequency variations for Models $\mathrm{A}-\mathrm{B}$ as functions of frequency. We only show those modes that are shown in Figure 1. From this figure we can see that a deep, strong magnetic field near the base of the convection zone tends to decrease the low- and medium-degree $p$-mode frequencies. The main reason for this is a change in the radius of the model caused by the magnetic fields. The decreases in frequency (as well as the large change in radius) are contrary to the observed changes. 


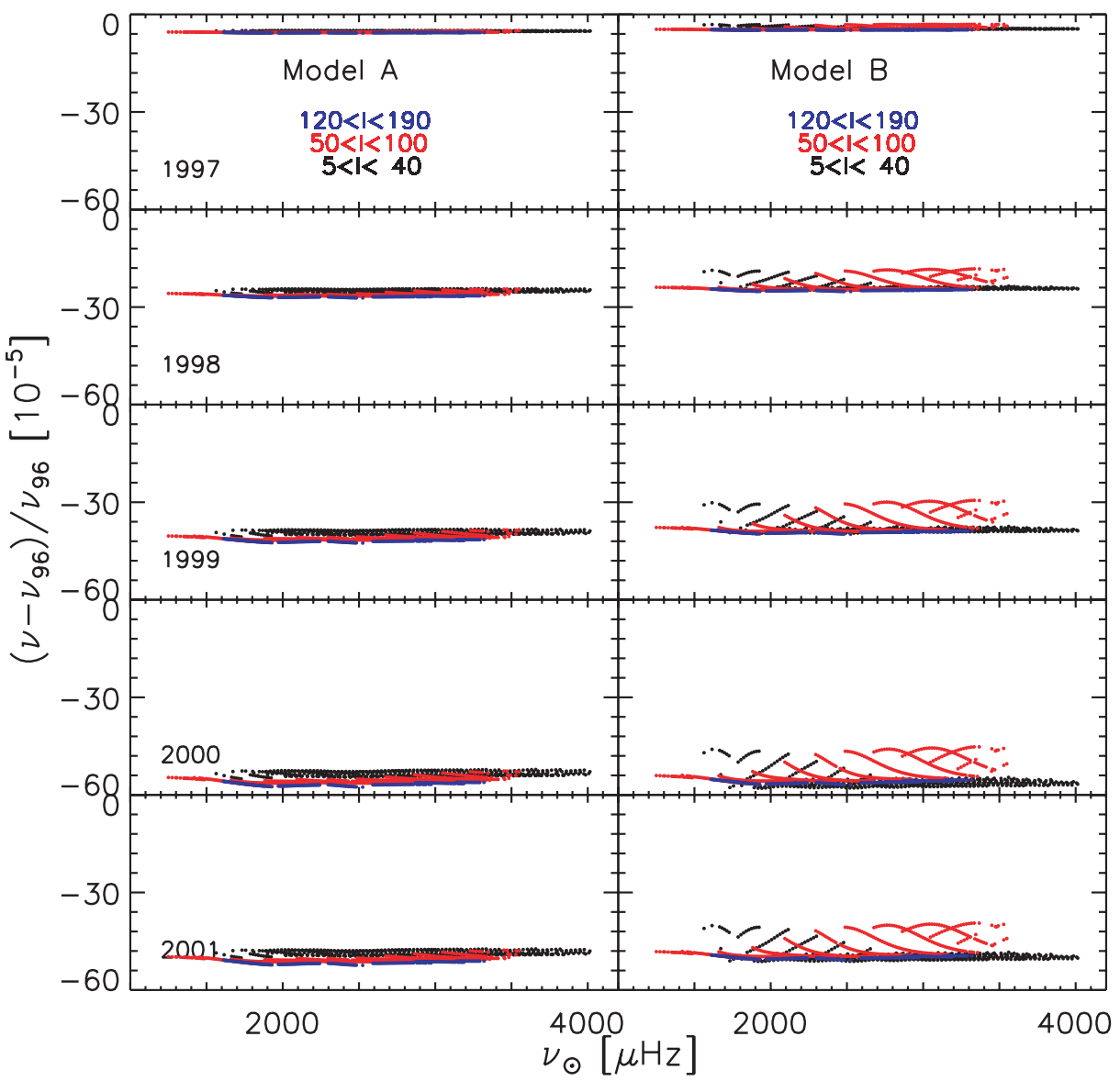

FIG. 8.-Calculated relative frequency variations for models A-B as functions of frequency

Models $\mathrm{C}-\mathrm{F}$ are better in that they show no change in the position of the base of the solar convection zone. However, the frequency changes do not match the observations. Figure 9 shows the calculated relative $p$-mode frequency variations for Models $\mathrm{C}$ to $\mathrm{F}$ as functions of frequency. We only show the low activity and activity maximum cases. A comparison of this figure with the 1997 and 2000 panels of Figure 1 shows that none of these models reproduce the observed changes in the Sun. The predominant effect is a decrease in the frequencies with time, which is not observed. The smaller scale details in the frequency differences in Figure 9 are due to localized changes in the sound-speed

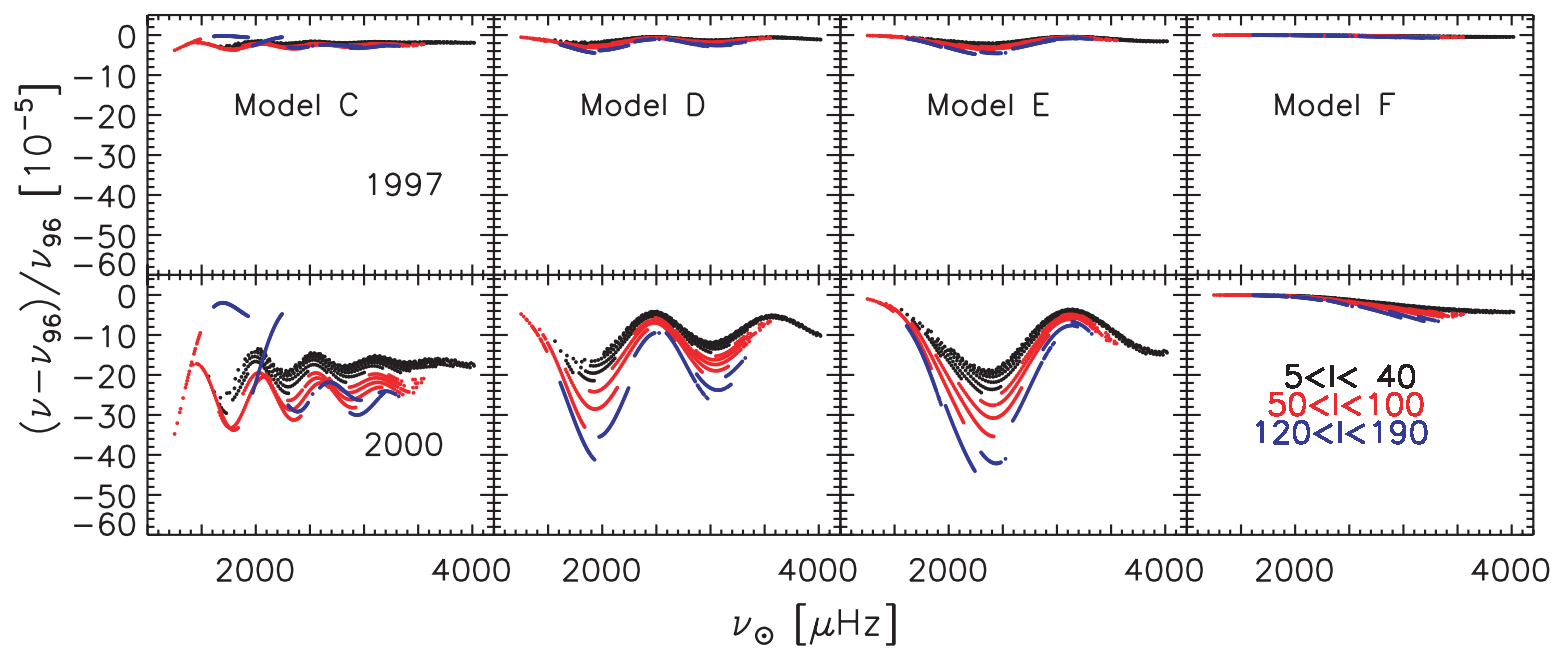

FIG. 9.-Calculated relative frequency variations for models $\mathrm{C}-\mathrm{F}$ as functions of frequency. These models have a Gaussian profile for the magnetic field. 
profiles of these modes caused by the localized magnetic fields. Only model F has frequency difference with a similar shape as that of the Sun, although the sign is wrong. The shape is dictated by the fact the magnetic field in Model F is confined to the surface layers, and hence the changes in structure are confined to the surface layers.

Another model in the same category, but with a different magnetic field profile is model M. This model too fails to satisfy the solar frequency constraints (see Fig. 10). Model $\mathrm{M}$ at least has the same shape of the frequency change as the observations (i.e., the frequency changes are a smooth function of frequency, which is a result of structural changes at the very outer layers), but again the sign is wrong.

These models (A to F, and M) seem to imply that this category of models do not satisfy helioseismic constraints. Even though we may get the same shape of the change in frequencies (model $\mathrm{F}$ and $\mathrm{M}$ ), in all cases the change in frequencies has the opposite sign. Model A and F imply that we need to confine the variable magnetic fields to the surface layers to get the correct frequency change profile, but need better physics to get the correct sign.

2. Models with partial effect of turbulence (models $G-J$ and $N$ ).-Figure 11 shows the calculated relative $p$-mode frequency variations for models $G$ to $J$ as functions of frequency. These are the models with weak turbulence, with magnetic field profiles very similar to models A to F. Again, except for model $\mathrm{J}$, which has the magnetic field confined to the surface, neither the shape nor the sign of the frequency changes match the observations. For model $\mathrm{J}$ the shape of the change is similar to the observations, but the sign is not. Model $\mathrm{N}$ falls in the same category of models as model $\mathrm{G}$ to $\mathrm{J}$, but has a different magnetic field profile, and the frequency changes of this model are shown in Figure 12. The shape of the frequency differences is similar to that of the Sun, but again the sign is incorrect. Therefore, these models show that introducing weak turbulence is not enough to obtain models that match helioseismic data.

3. Fully turbulent models (models $K, L, O$, and P).Figures 13 and 14 show the calculated relative $p$-mode frequency variations for models $\mathrm{K}, \mathrm{L}, \mathrm{O}$, and $\mathrm{P}$ as functions of frequency. Models $\mathrm{K}$ and $\mathrm{L}$ differ from models $\mathrm{O}$ and $\mathrm{P}$ in that their magnetic filed profiles are different. Again the frequency changes have the opposite sign of that observed. In addition, these models do not match the irradiance change and temperature change data either, so clearly we are still doing something wrong.

4. Models with magnetically modulated turbulence.Figure 15 shows the calculated relative $p$-mode frequency variations for these two models are quite similar. Defining

$$
\chi^{2}=\frac{1}{5} \sum_{\text {year }} \frac{1}{N} \sum_{p \text {-mode }}\left(\frac{\Delta \nu_{\odot} / \nu_{\odot}-\Delta \nu / \nu}{\sigma}\right)^{2}
$$

as $\chi^{2}$ per-degree-of-freedom. We have $\chi^{2}=20$ for model

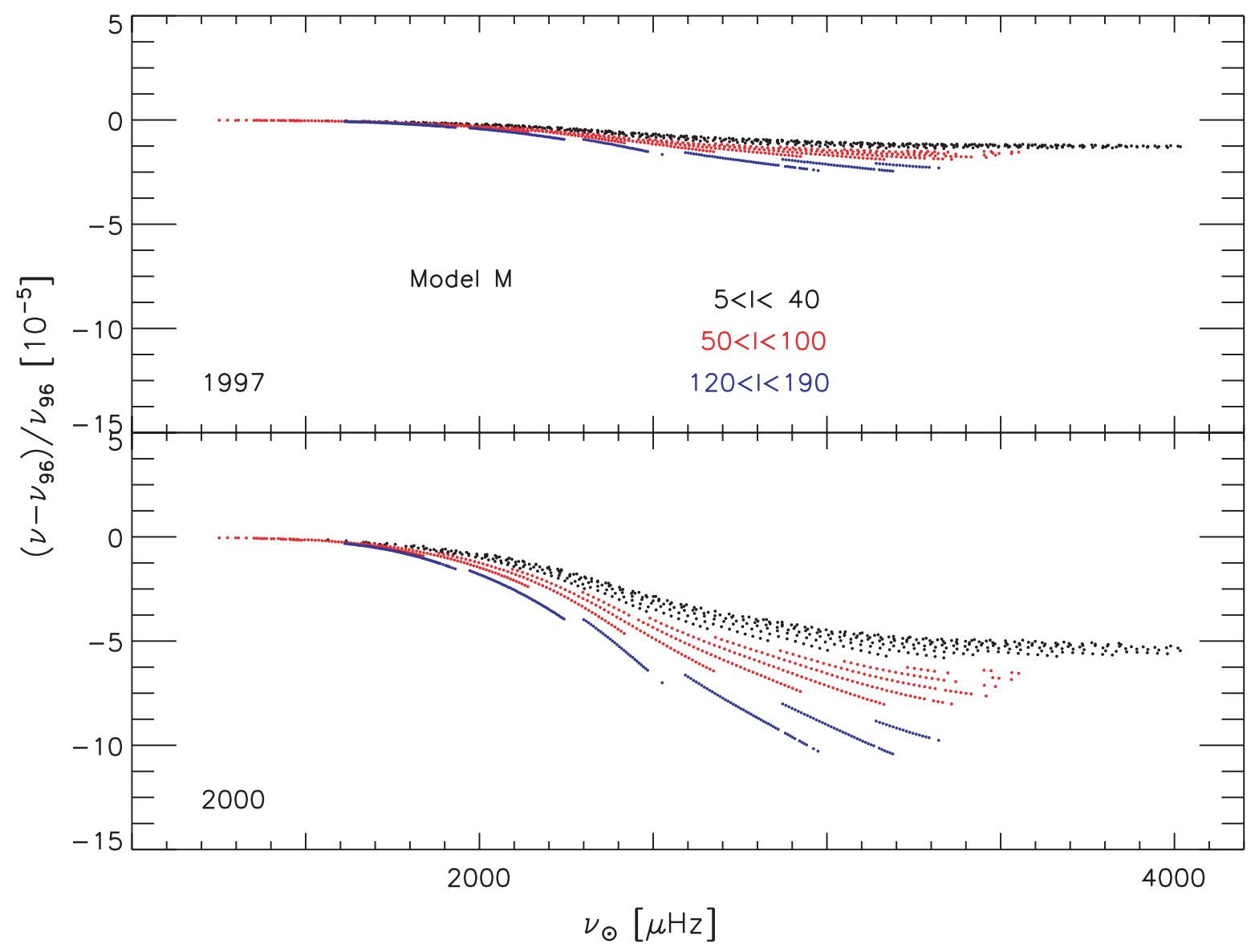

FIG. 10. - Calculated relative frequency variations for model $\mathrm{M}$ as a function of frequency. The magnetic field has the same profile as the turbulent kinetic energy. 


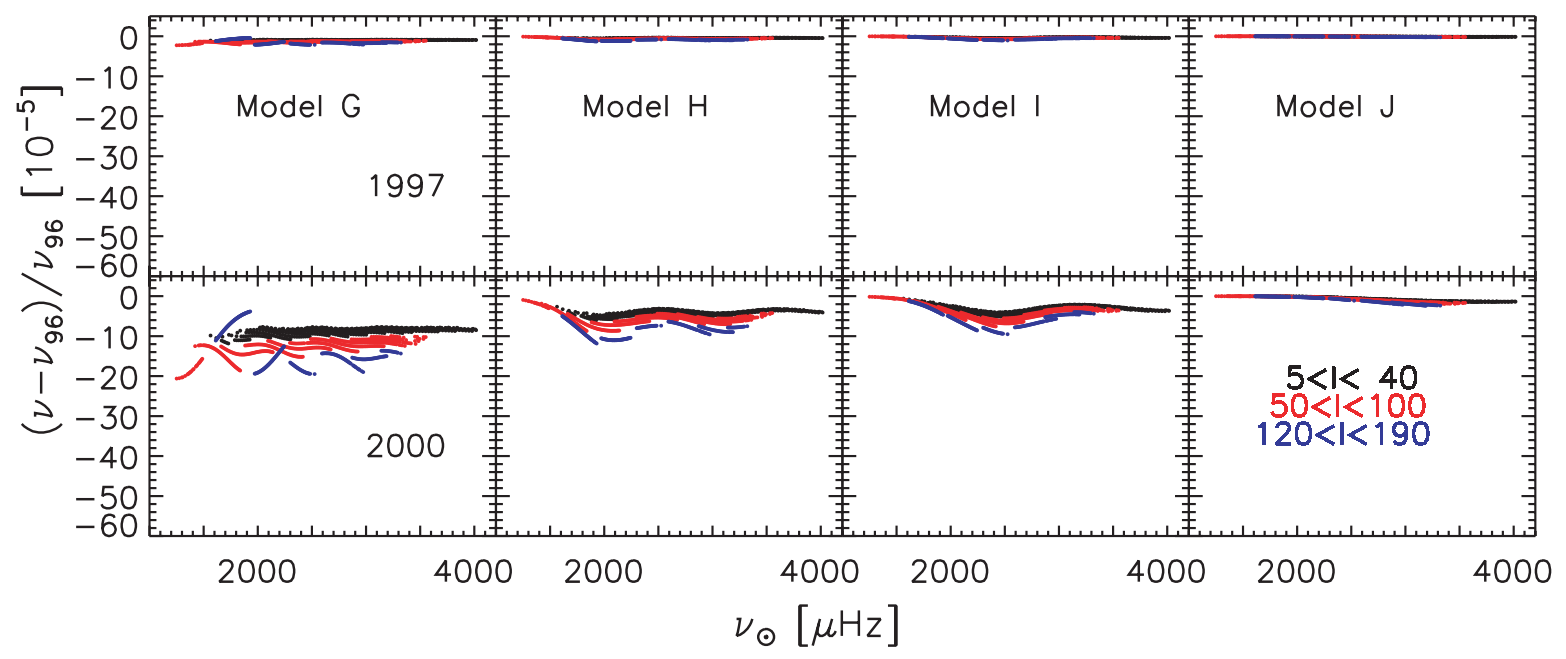

FIG. 11.-Calculated relative frequency variations for models G-J as functions of frequency. These models have a Gaussian profile for the magnetic field.

$\mathrm{Q}$ and $\chi^{2}=7$ for model $\mathrm{R}$. In contract to these models, model A gives a $\chi^{2}$ of 4318. Model $\mathrm{Q}$, in addition to having a higher $\chi^{2}$, does not satisfy the luminosity change and temperature change constraints. Thus, model $\mathrm{R}$ is the best we have. Thus, it appears that the only way we can get a matching model is not only to include a full treatment of turbulence but also to have the turbulence be modulated by the magnetic field. This in itself is not surprising since magnetic flux tubes are known to suppress convection.

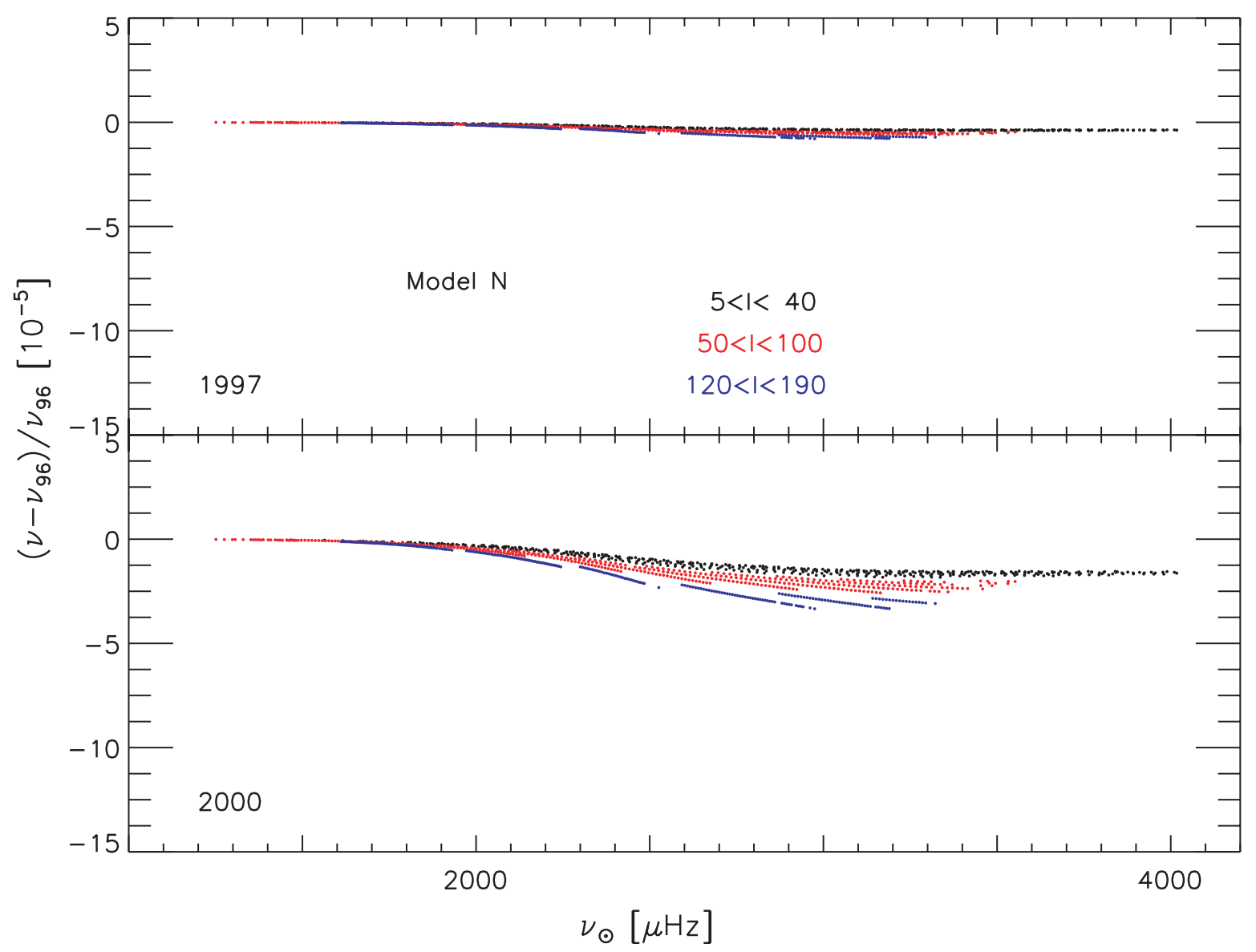

FIg. 12.-Calculated relative frequency variations for model $\mathrm{N}$ as a function of frequency. The magnetic field has the same profile as the turbulent kinetic energy. 


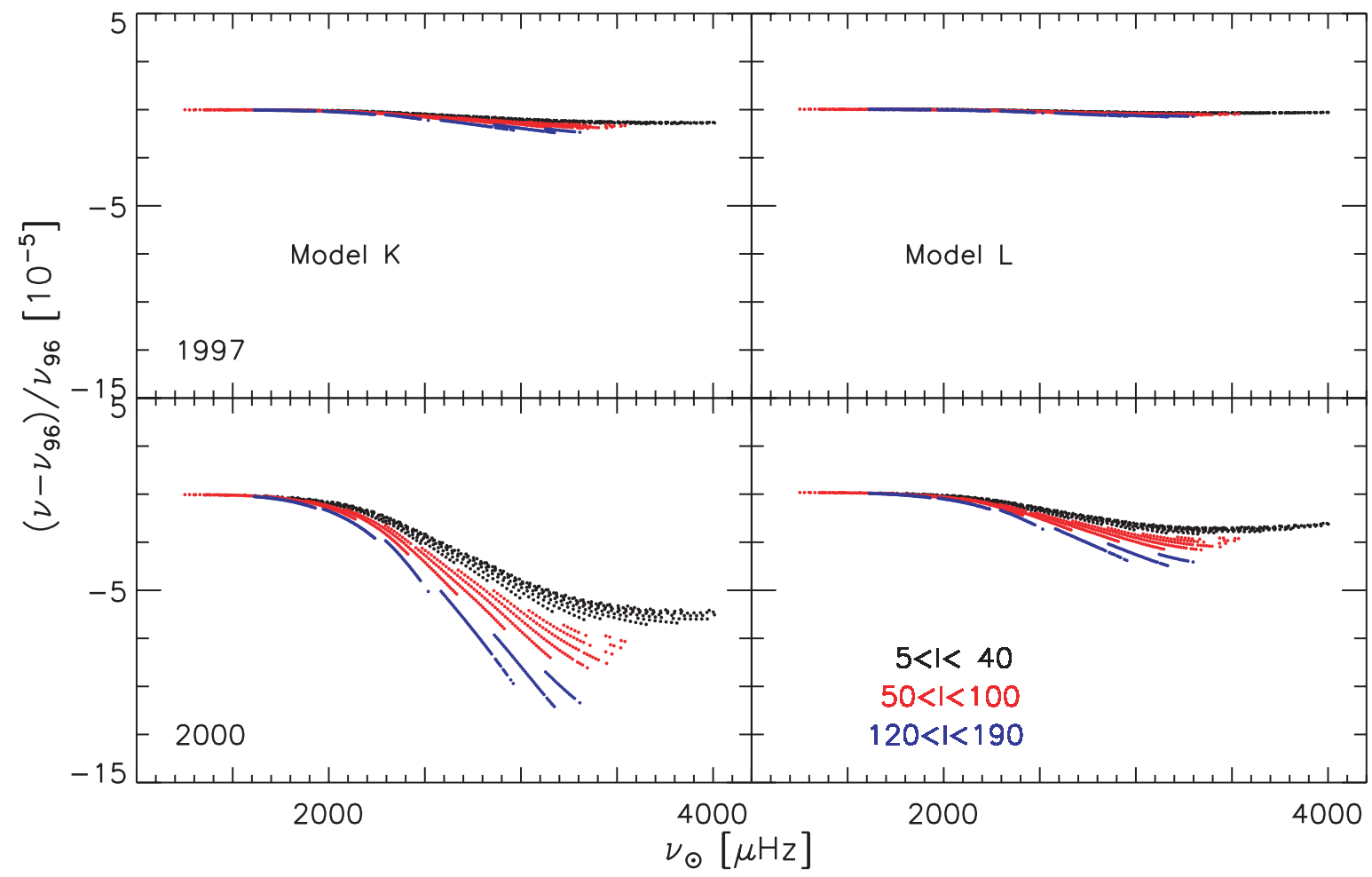

FIG. 13.-Calculated relative frequency variations for models $\mathrm{K}-\mathrm{L}$ as functions of frequency. These two models have a Gaussian profile for the magnetic field.

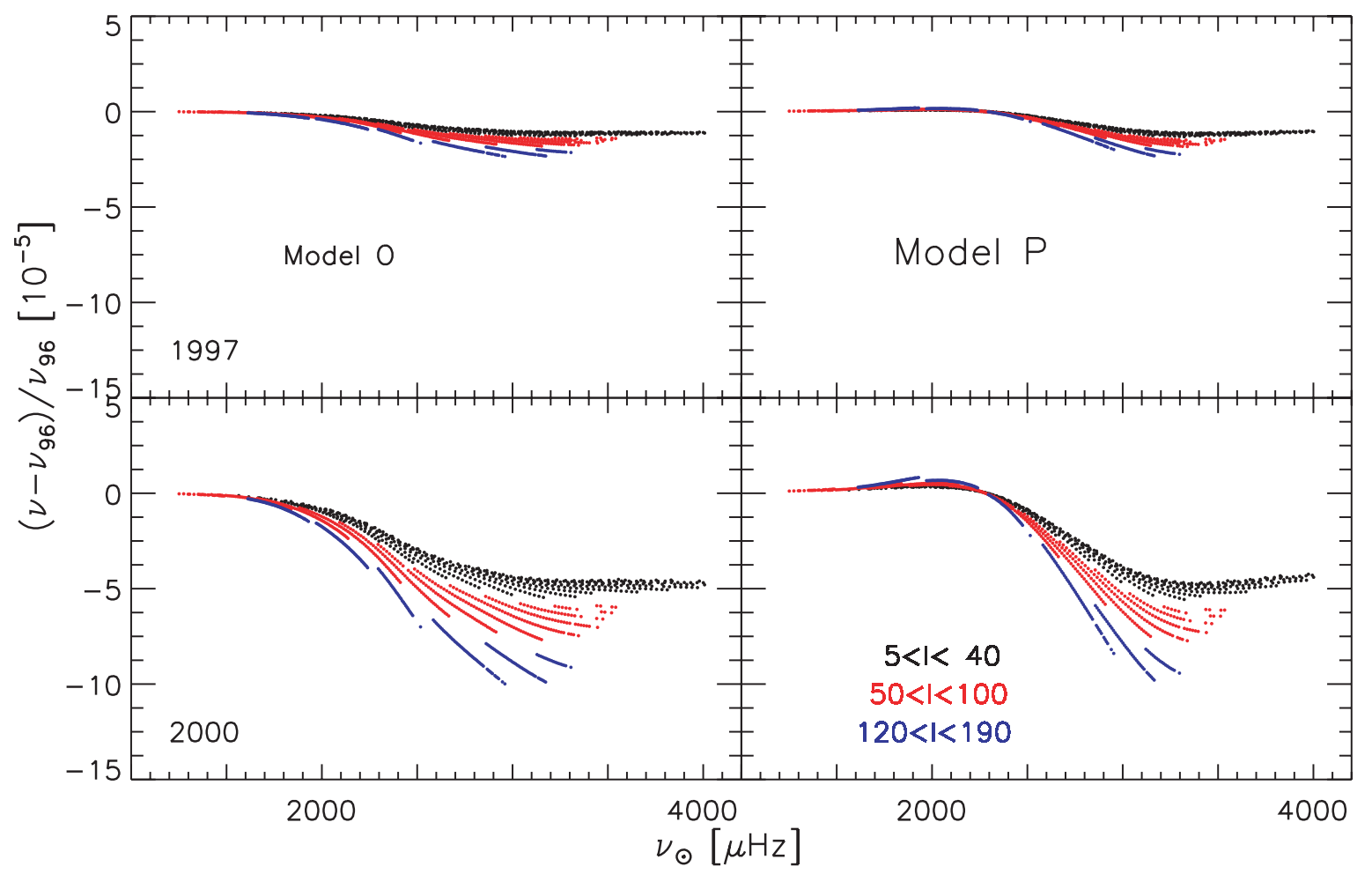

FIG. 14.-Calculated relative frequency variations for models O-P as functions of frequency. The magnetic field has the same profile as the turbulent kinetic energy. 


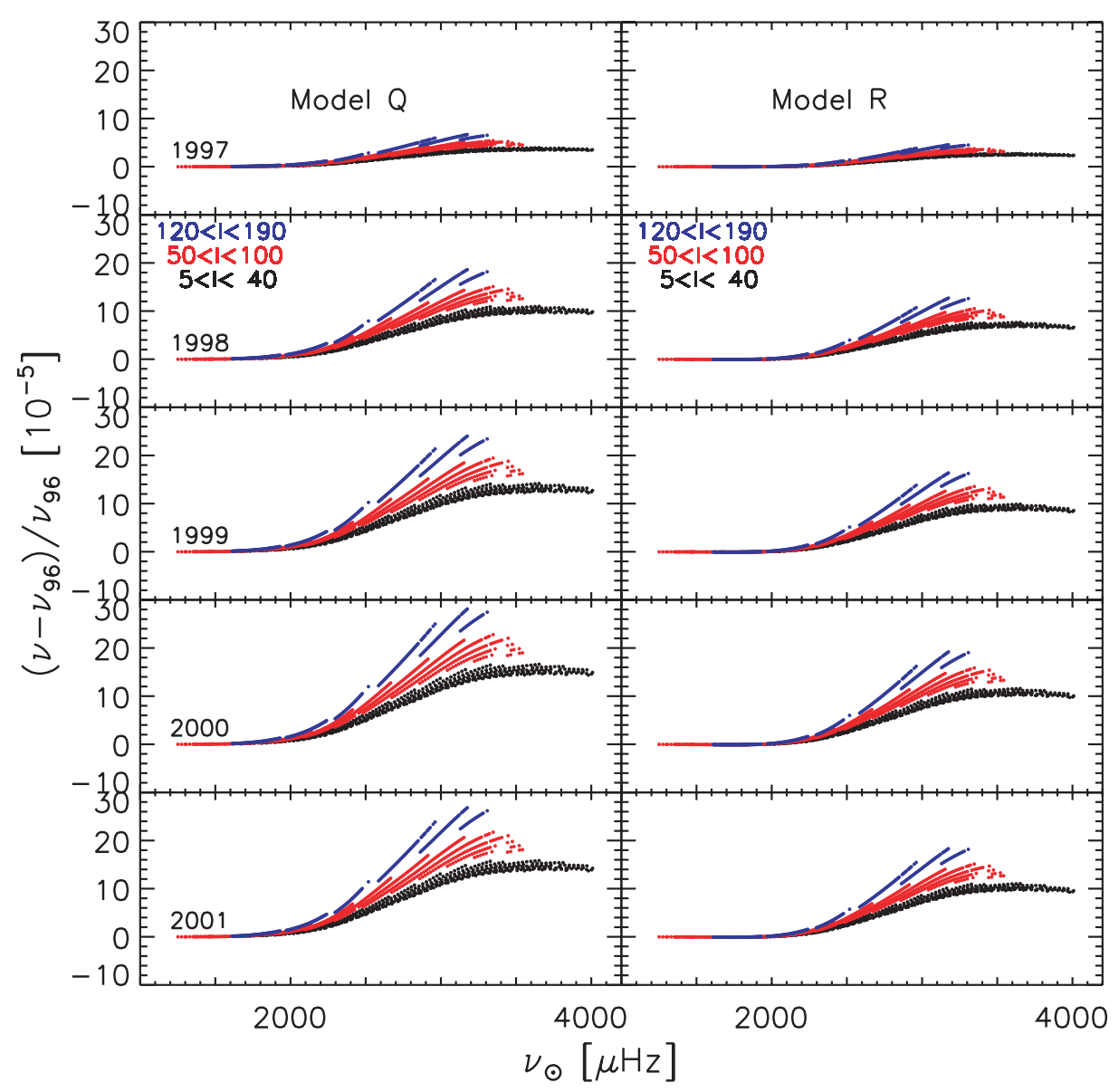

FIG. 15.-Calculated relative frequency variations for models $\mathrm{Q}-\mathrm{R}$ as functions of frequency

In summary, only models $\mathrm{Q}$ and $\mathrm{R}$ can pass all the helioseismic tests, but we still need further work.

\section{DISCUSSION AND CONCLUSIONS}

Most previous work in this field focus on explaining the TSI, and a few venture into $W$. In this paper we test our models with all relevant observations, i.e., TSI, photospheric temperature, radius, and heliosesimic constraints. The presence of time-varying magnetic fields in the solar interior causes changes in the structure of the Sun, which manifest themselves in the variations of all global and helioseismic parameters. The global parameters alone, if known precisely, can uniquely constrain the properties of the internal magnetic field. In that case, helioseismic observations could be used as independent tests of the models. However, because the observations of most global parameters are still somewhat uncertain, we are compelled to use helioseismological data as part of our model fitting.

A summary of how the different models fare when compared with the observational constraints is given in Table 3 . An entry of " $y$ " means a favorable comparison, and " $n$ " is an unfavorable one. Because we assume that the solar irradiance is proportional to luminosity only, we call the irradiance variations $\Delta L$. In the case of the radius constraint, since the amount of change is not certain, but the sign of the change seems to be, we assume that the observations are matched by all models that show a decrease in the
TABLE 3

Summary of Comparison of Models With ObServations

\begin{tabular}{|c|c|c|c|c|c|c|}
\hline \multirow[b]{2}{*}{ Model } & \multirow[b]{2}{*}{ GROUP } & \multicolumn{5}{|c|}{ Observational Constraints } \\
\hline & & $\Delta L$ & $\Delta T$ & $\Delta R$ & $\Delta R_{\mathrm{CZ}}$ & $\Delta \nu$ \\
\hline \multicolumn{7}{|c|}{ Models with a Gaussian $\boldsymbol{B}$ Profile } \\
\hline A & I & $\mathrm{y}$ & $\mathrm{n}$ & $\mathrm{n}$ & $\mathrm{n}$ & $\mathrm{n}$ \\
\hline $\mathrm{B} \ldots \ldots \ldots \ldots \ldots \ldots \ldots \ldots \ldots \ldots \ldots \ldots$ & I & $\mathrm{y}$ & $\mathrm{n}$ & $\mathrm{n}$ & $\mathrm{n}$ & $\mathrm{n}$ \\
\hline $\mathrm{C} \ldots \ldots \ldots \ldots \ldots \ldots \ldots \ldots$ & I & $\mathrm{y}$ & $\mathrm{y}$ & $\mathrm{n}$ & $\mathrm{y}$ & $\mathrm{n}$ \\
\hline $\mathrm{D}$ & I & $\mathrm{y}$ & $\mathrm{y}$ & $\mathrm{n}$ & $\mathrm{y}$ & $\mathrm{n}$ \\
\hline $\mathrm{E}$ & I & $\mathrm{y}$ & $\mathrm{y}$ & $\mathrm{n}$ & $\mathrm{y}$ & $\mathrm{n}$ \\
\hline $\mathrm{F} \ldots \ldots \ldots \ldots \ldots \ldots \ldots$ & I & $\mathrm{n}$ & $\mathrm{n}$ & $\mathrm{n}$ & $\mathrm{y}$ & $\mathrm{n}$ \\
\hline $\mathrm{G}$ & II & $\mathrm{y}$ & $\mathrm{y}$ & $\mathrm{n}$ & $\mathrm{y}$ & $\mathrm{n}$ \\
\hline Н ....................... & II & $\mathrm{y}$ & $\mathrm{y}$ & $\mathrm{n}$ & $\mathrm{y}$ & $\mathrm{n}$ \\
\hline I ...................... & II & $\mathrm{y}$ & $\mathrm{y}$ & $\mathrm{n}$ & $\mathrm{y}$ & $\mathrm{n}$ \\
\hline $\mathrm{J}$ & II & $\mathrm{n}$ & $\mathrm{n}$ & $\mathrm{n}$ & $\mathrm{y}$ & $\mathrm{n}$ \\
\hline K & III & $\mathrm{n}$ & $\mathrm{n}$ & $\mathrm{n}$ & $\mathrm{y}$ & $\mathrm{n}$ \\
\hline L ..................... & III & $\mathrm{n}$ & $\mathrm{n}$ & $\mathrm{y}$ & $\mathrm{y}$ & $\mathrm{n}$ \\
\hline
\end{tabular}

Models with $\boldsymbol{B}$ Profile from Turbulence

\begin{tabular}{|c|c|c|c|c|c|c|}
\hline М & I & $\mathrm{n}$ & $\mathrm{n}$ & $\mathrm{n}$ & $\mathrm{y}$ & $\mathrm{n}$ \\
\hline $\mathrm{N}$ & II & $\mathrm{n}$ & $\mathrm{n}$ & $\mathrm{n}$ & $\mathrm{y}$ & $\mathrm{n}$ \\
\hline $\mathrm{O}$ & III & $\mathrm{n}$ & $\mathrm{n}$ & $\mathrm{n}$ & $\mathrm{y}$ & $\mathrm{n}$ \\
\hline 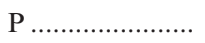 & III & $\mathrm{n}$ & $\mathrm{n}$ & $\mathrm{n}$ & $\mathrm{y}$ & $\mathrm{n}$ \\
\hline Q & IV & $\mathrm{n}$ & $\mathrm{n}$ & $\mathrm{y}$ & $\mathrm{y}$ & $\mathrm{y}$ \\
\hline R....................... & IV & $\mathrm{y}$ & $\mathrm{y}$ & $\mathrm{y}$ & $\mathrm{y}$ & $\mathrm{y}$ \\
\hline
\end{tabular}

Note.-An entry of " $y$ " means a favorable comparison, and " $n$ " is an unfavorable one. 
TABLE 4

Helioseismic Models

\begin{tabular}{|c|c|c|c|c|c|c|c|}
\hline $\begin{array}{c}\text { Model } \\
\text { (1) }\end{array}$ & $\begin{array}{c}B_{96} \\
\left(10^{5} \mathrm{G}\right) \\
(2)\end{array}$ & $\begin{array}{c}B_{00} \\
\left(10^{5} \mathrm{G}\right) \\
(3)\end{array}$ & $\begin{array}{c}\text { Thickness } \\
\left(R_{\odot}\right) \\
(4)\end{array}$ & $\begin{array}{c}\Delta \ln L \\
\left(10^{-3}\right) \\
(5)\end{array}$ & $\begin{array}{c}\Delta \ln R \\
\left(10^{-6}\right) \\
(6)\end{array}$ & $\begin{array}{c}\Delta \ln T \\
\left(10^{-6}\right) \\
(7)\end{array}$ & $\begin{array}{c}\Delta \ln R_{\mathrm{CZ}} \\
\left(10^{-4}\right) \\
(8)\end{array}$ \\
\hline $\mathrm{H} 1 \ldots \ldots \ldots \ldots \ldots \ldots$ & 3.0 & 18 & 0.05 & 1.1 & 412 & 62.8 & -1150 \\
\hline ......... & 5.0 & 19 & 0.05 & 1.1 & 436 & 50.7 & -1170 \\
\hline $\mathrm{H} 3 \ldots \ldots \ldots \ldots \ldots \ldots$ & 3.0 & 3.5 & 0.05 & 0.02 & 6 & 1 & 6.5 \\
\hline .............. & 5.0 & 5.7 & 0.05 & 0.03 & 10 & 3 & 6.5 \\
\hline
\end{tabular}

radius as activity increases. As can be seen from Table 3, most models fail to reproduce all observations. Satisfying the current global constraints is easier than satisfying the helioseismological constraints, mainly because of the poor quality former and the high precision of the latter. While for many of our models (F, J, M-P) the shape of the frequency changes is the same as the observed changes, the sign of the change is opposite of what is observed. Only model R satisfies all constraints.

Our successful models suggest that turbulence near the surface of the Sun must play a key role in solar variability, at least over the timescale of a solar cycle. Model R includes the effects of magnetic fields and turbulence and the feedback between them. Models with only magnetic fields do not reproduce all the observations. Qualitatively, the interaction between magnetic fields and turbulence is simple: magnetic fields tend to inhibit turbulence. However, the quantitative behavior of this interaction is not known, and we are forced to introduce parameters to describe this interaction. Thus, in some ways we have simplified models. A quantitative description of the effect of turbulence on magnetic fields and vice versa should be obtainable from numerical simulations.

Conventionally, the solar dynamo is supposed to be located in the tachocline. The tachocline is the thin region at the base of the convection zone where the strong differential rotation in the convection zone changes into solid-body type rotation in the interior. Helioseismic observation give a fairly strong upper limit to the magnetic field in those regions (<0.3 MG [Basu 1997]; 0.4-0.7 MG [Chou \& Serebryanskiy 2002]). To show that such a field is not enough to explain solar variability, we have constructed four models with the magnetic field configuration as suggested from helioseismic studies. Models $\mathrm{H} 1$ and $\mathrm{H} 2$ are constructed assuming that the models satisfy the irradiance change constraint. Models $\mathrm{H} 3$ and $\mathrm{H} 4$ were constructed to see how much the magnetic field at the base of the $\mathrm{CZ}$ could change without changing the position of the base of the CZ. The models and their results are summarized in Table 4. Column (1) is the name of the model, column (2) is the magnetic field at the solar minimum (1996), column (3) is the field in year 2000, column (4) is the $\sigma$ of the field distribution (assumed to be a Gaussian), and columns (5), (6), (7), and (8) are changes in luminosity, radius, temperature, and position of the $\mathrm{CZ}$ base, respectively. We can see that the $\mathrm{CZ}$ base changes for models $\mathrm{H} 1$ and $\mathrm{H} 2$ are well above what is observed. Besides, the final magnetic field at the base of the $\mathrm{CZ}$ is also larger than the helioseismic estimates. The frequency changes do not match the observations either (see Fig. 16). Models $\mathrm{H} 3$ and $\mathrm{H} 4$ on the other hand, fail to satisfy any of the global criteria. They also fail to reproduce the frequency changes (see Fig. 17). As far as the frequency changes are concerned, model $\mathrm{H} 3$ yields a $\chi^{2}$ per degree of freedom of 128 . Thus, while there is likely to be a small magnetic field at the base of the convection zone, it is not enough to explain the various observations. This field could of course provide the seed fields that are then enhanced by turbulence in the upper layers. A variable field a the CZ base may also be responsible for longer timescale variability that affects the Earth's climate (see Sofia \& Li 2001).

The present work has one major limitation: the current models are one-dimensional. This means that we can only use a shell-like magnetic configuration in the spherical approximation. In this case, any entropy perturbation has to penetrate the magnetic shell. This rules out the possibility for an energy flux or a flow to go around the magnetic regions. Therefore, we cannot use the latitudinal change information to explore the latitudinal distribution of magnetic fields in the interior of the Sun. In order to make sure that our conclusions are robust, we have to generalize our models to at least two dimensions. This limitation notwithstanding, the fact that only one of our models can satisfy observations tells us something fundamental about the physical processes that affect solar cycle-related changes. We find that the interaction between magnetic fields and turbulence is essential in explaining the observations we currently have. We are currently in the process of developing codes to construct two-dimensional solar models that would help us investigate the effects of more realistic magnetic field configurations.

This work was supported by NASA grant 899-10633 and NSF grants ATM 9303023 and ATM 0206130. This work utilizes data from the Solar Oscillations Investigation/ Michelson Doppler Imager (SOI/MDI) on the Solar and Heliospheric Observatory $(\mathrm{SOHO})$. SOHO is a project of international cooperation between ESA and NASA. MDI is supported by NASA grants NAG5-8878 and NAG5-10483 to Stanford University. 


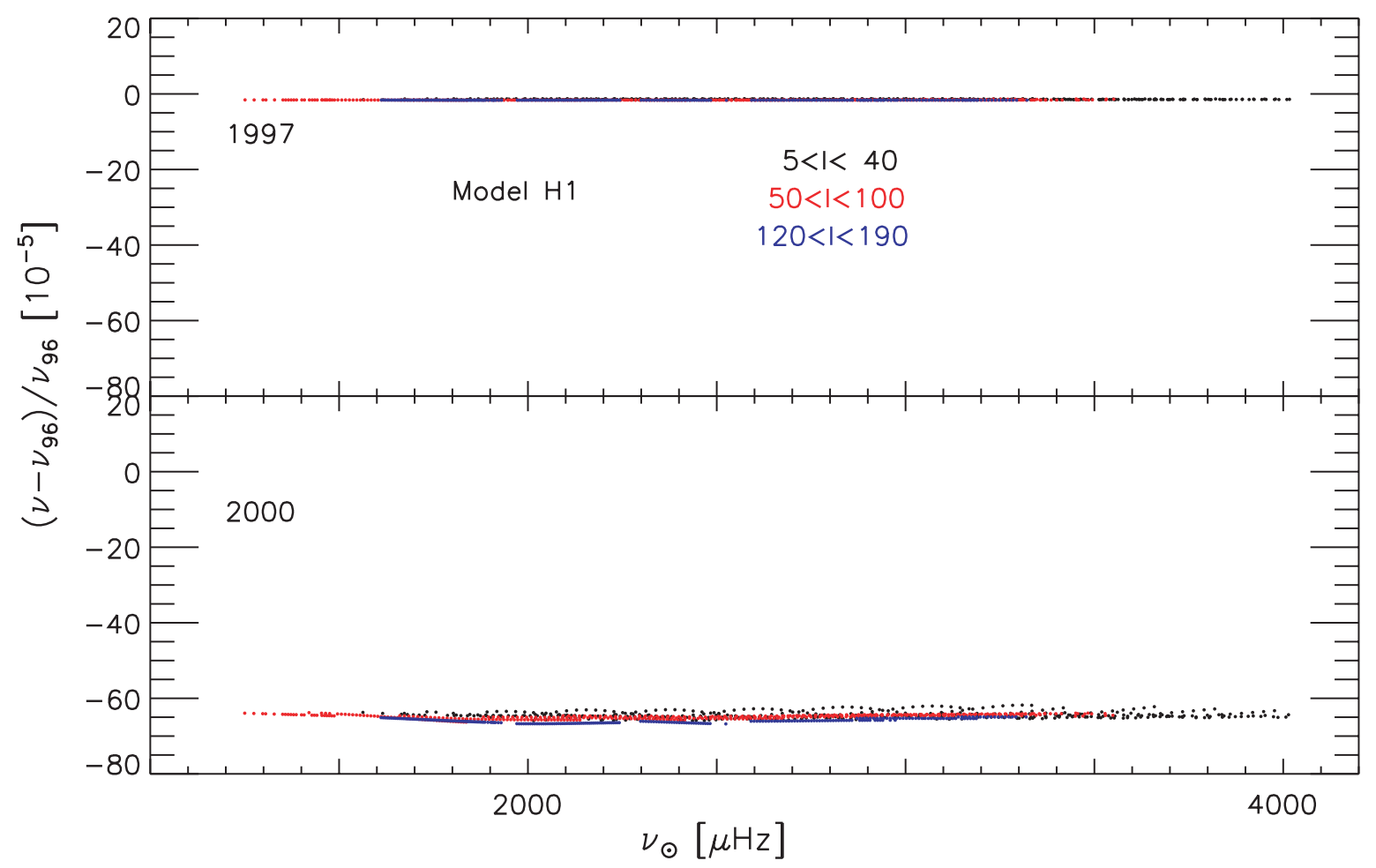

FIG. 16. - Calculated relative frequency variations for model H1 as a function of frequency

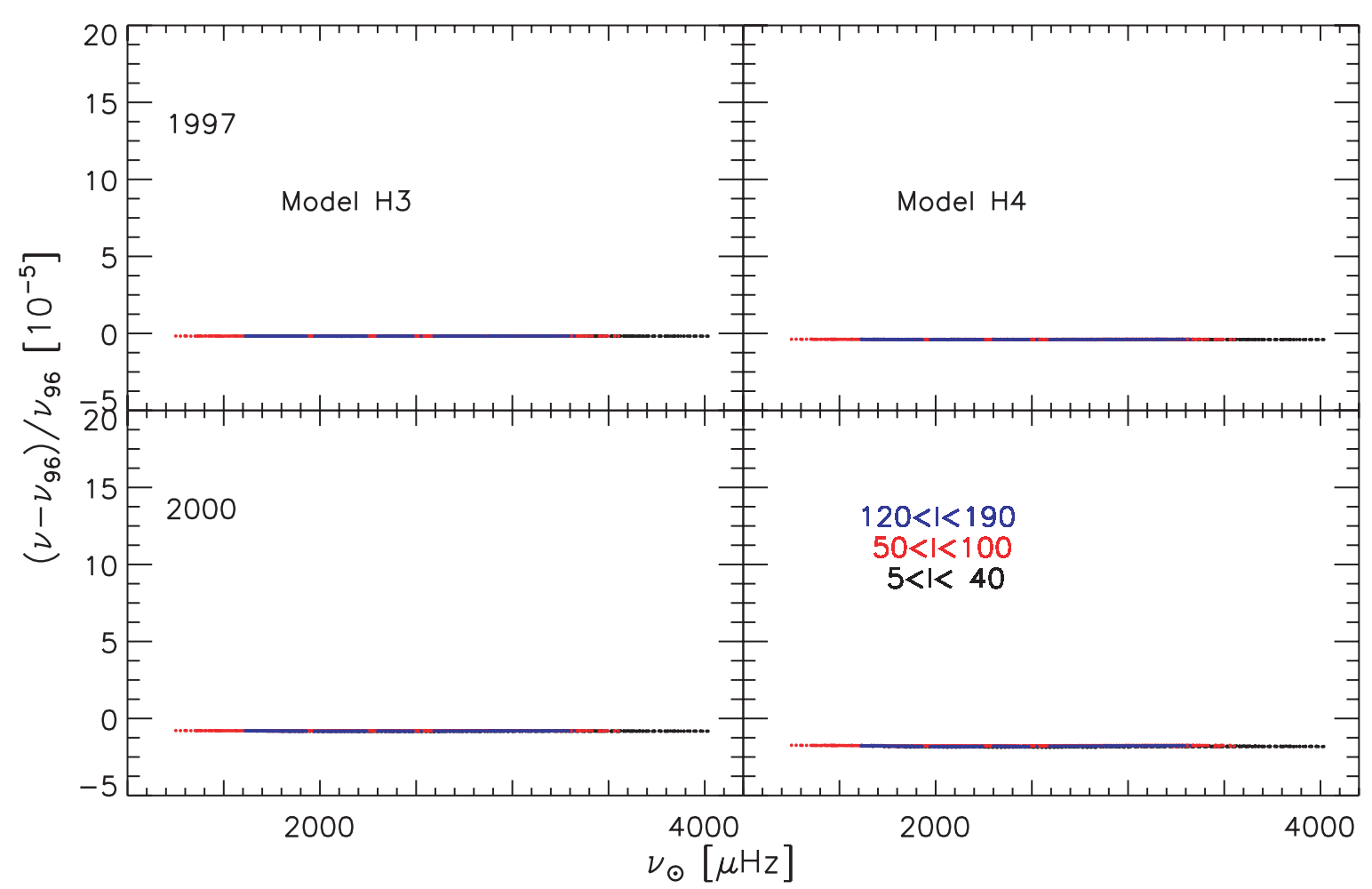

FIG. 17.-Calculated relative frequency variations for models $\mathrm{H} 3$ and $\mathrm{H} 4 \mathrm{as}$ functions of frequency 
Alexander, D. R , \& Ferguson, J. W. 1994, ApJ, 437, 879

Antia, H. M. 1998, A\&A, 330, 336

Antia, H. M., Basu, S., Pintar, J., \& Pohl, B. 2000, Sol. Phys., 192, 459

Antia, H. M., Basu, S., Pintar, J., \& Schou, J. 2001, in Proc. SOHO 10/

GONG 2000 Workshop: Helio- and Asteroseismology at the Dawn of the Millennium, ed. A. Wilson (ESA SP-464; Noordwijk: ESA), 27

Asplund, M., Ludwig, H.-G., Nordlund, A., \& Stein, R. F. 2000, A\&A, 359,669

Basu, S. 1997, MNRAS, 288, 572

. 2002, in Proc. SOHO 11 Workshop, From Solar Minimum to Maximum: Half a Solar Cycle with $\mathrm{SOHO}$, ed. A. Wilson (ESA SP-508; Noordwijk: ESA), 7

Basu, S., \& Antia, H. M. 1997, MNRAS, 287, 189

2000, Sol. Phys., 192, 449

2002 , in Proc. SOHO 11 Workshop, From Solar Minimum to

Maximum: Half a Solar Cycle with $\mathrm{SOHO}$, ed. A. Wilson (ESA SP-508;

Noordwijk: ESA), 59

Basu, S., Pinsonneault, M. H., \& Bahcall, J. N. 2000, ApJ, 529, 1084

Bond, G., et al. 2001, Science, 294, 2130

Caccin, B., \& Penza, V. 2002, A\&A, 386, 286

Callen, H. B. 1966, Thermodynamics (New York: John Wiley), 242

Cattaneo, F. 1999, ApJ, 515, L39

Christensen-Dalsgaard, J., Gough, D. O., \& Thompson, M. J. 1991, ApJ, 378,413

Chou, D.-Y., \& Serebryanskiy, A. 2002, ApJ, 578, L157

Dearborn, D. S. P., \& Blake, J. B. 1980a, Nature, 287, 365 1980b, ApJ, 237, 616 1980b, ApJ, 237, 616

Deupree, R. G. 1990, ApJ, 357, 175 1995, ApJ, 439, 357 1998, ApJ, 499, 340 2001 A J J 552, 268

Dziembowski, W. A., Goode, P. R., di Mauro, M. P., Kosovichev, A. G., \& Schou, J. 1998, ApJ, 509, 456

Dziembowski, W. A., Goode, P. R., Kosovichev, A. G., \& Schou, J. 2000, ApJ, 537, 1026

Dziembowski, W. A., Goode, P. R., \& Schou, J. 2001, ApJ, 553, 897

Eddy, J. A. 1976, Science, 192, 1189

Egidi, A., Caccin, B., Berrilli, F., \& Sofia, S. 1999, in Magnetic Fields and Solar Processes, ed. A. Wilson (ESA SP-448; Noordwijk: ESA:) 15

Emilio, M., Kuhn, J. R., Bush, R. I., \& Scherrer, P. 2000, ApJ, 543, 1007

Endal, A. S., Sofia, S., \& Twigg, L. W. 1985, ApJ, 290, 748

Fox, P. A., Theobald, M. L., \& Sofia, S. 1991, ApJ, 383, 860

Fröhlich, C. 2000, Space Sci. Rev., 94, 15

Gray, D. F., \& Livingston, W. C. 1997a, ApJ, 474, 798 1997b, ApJ, 474, 802

\section{EFERENCES}

Guenther, D. B. 1994, ApJ, 422, 400

Guenther, D. B., \& Demarque, P. 1997, ApJ, 484, 937

Iglesias, C. A., \& Rogers, F. J. 1996, ApJ, 464, 943

Krishna Swamy, K. S. 1966, ApJ, 145, 174

Kuhn, J. R. 1996, in Global Changes in the Sun, ed. T. Roca-Cortés Cambridge: Cambridge Univ. Press), 231

. 2003, Adv. Space Res., in press

Kuhn, J. R., \& Armstrong, J. D. 2003, in Solar Variability and Its Influence on the Earth's Atmosphere and Climate System, ed. J. M. Pap et al. (Geophys. Monogr.; Washington, DC: AGU), in press

Kuhn, J. R., Bush, R., Scheick, X., \& Scherrer, P. 1998, Nature, 392, 155

Lean, J. L., Cook, J., Maquette, W., \& Johannesson, A. 1998, ApJ, 492, 390

Li, L. H., Robinson, F. J., Demarque, P., Sofia, S., \& Guenther, D. B. 2002 , ApJ, 567, 1192

Li, L. H., \& Sofia, S. 2001, ApJ, 549, 1204

Lydon, T. J., \& Sofia, S. 1995, ApJS, 101, 357

Muller, R. 1986, Sol. Phys., 119, 229

Robinson, F. J., Demarque, P., Li, L. H., Sofia, S., Kim, Y. C., Chan, K. L., \& Guenther, D. B. 2003, MNRAS, 340, 923

Rogers, F. J., Swenson, F. J., \& Iglesias, C. A. 1996, ApJ, 456, 902

Rosenthal, C. S., Christensen-Dalsgaard, J., Nordlund, A, Stein, R. F., \& Trampedach, R. 1999, A\&A, 351, 689

Schou, J., Kosovichev, A. G., Goode, P. R., \& Dziembowski, W. A. 1997, ApJ, 489, L197

Sharma, M. 2002, Earth Planet. Sci. Lett., 199, 459

Shindell, D. T., Schmidt, G. A., Mann, M. E., Rind, D., \& Waple, A. 2001, Science, 294, 2149

Sofia, S., Heaps, W., \& Twigg, L. W. 1994, ApJ, 427, 1048

Sofia, S., \& Li, L. H. 2001, J. Geophys. Res., 106, 12,969

. 2003, in Solar Variability and Its Influence on the Earth's Atmosphere and Climate System, ed. J. M. Pap et al. (Geophys. Monogr.; Washington, DC: AGU), in press

Sofia, S., O'Keefe, J., Lesh, J. R., \& Endal, A. S. 1979, Science, 204, 1306

Stein, R. F., \& Nordlund, A. 1998, ApJ, 499, 914 2000, Sol. Phys., 192, 91

Title, A. M., Topka, K. P., Tarbell, T. D., Schmidt, W., Balke, C., \& Scharmer, G. 1992, ApJ, 393, 782

Tobias, S. M., Brummell, N. H., Clune, T. L., \& Toomre, J. 2001, ApJ, 549, 1183

Turcotte, S., et al. 2001, in IAU Colloq. 185, Radial and Nonradial Pulsations as Probes of Stellar Physics, ed. C. Aerts, T. R. Bedding, \& J. Christensen-Dalsgaard (San Francisco: ASP), 258

Ulrich, R. K. 1975, Science, 190, 619

Wilson, R. C., \& Hudson, H. S. 1991, Nature, 351, 42

Winnick, R. A., Demarque, P., Basu, S., \& Guenther 2002, ApJ, 576, 1075 